Journal for ImmunoTherapy of Cancer

\title{
Optimizing therapeutic outcomes of immune checkpoint blockade by a microbial tryptophan metabolite
}

\author{
Giorgia Renga (D) , ${ }^{1}$ Emilia Nunzi, ${ }^{1}$ Marilena Pariano, ${ }^{1}$ Matteo Puccetti, ${ }^{2}$ \\ Marina Maria Bellet, ${ }_{1}$ Giuseppe Pieraccini (D) , ${ }^{3}$ Fiorella D'Onofrio, ${ }^{1}$ Ilaria Santarelli, ${ }^{1}$ \\ Claudia Stincardini, ${ }^{1}$ Franco Aversa, ${ }^{4}$ Francesca Riuzzi, ${ }^{1}$ Cinzia Antognelli, ${ }^{1}$ \\ Marco Gargaro, ${ }^{1}$ Oxana Bereshchenko, ${ }^{5}$ Maurizio Ricci, ${ }^{2}$ Stefano Giovagnoli, ${ }^{2}$ \\ Luigina Romani, ${ }^{1}$ Claudio Costantini ${ }^{1}$
}

To cite: Renga G, Nunzi E, Pariano M, et al. Optimizing therapeutic outcomes of immune checkpoint blockade by a microbial tryptophan metabolite. Journal for ImmunoTherapy of Cancer 2022;10:e003725. doi:10.1136/ jitc-2021-003725

- Additional supplemental material is published online only. To view, please visit the journal online (http://dx.doi.org/10. 1136/jitc-2021-003725).

GR and EN are joint first authors Accepted 01 January 2022

\section{Check for updates}

(c) Author(s) (or their employer(s)) 2022. Re-use permitted under CC BY-NC. No commercial re-use. See rights and permissions. Published by BMJ.

${ }^{1}$ Department of Medicine and Surgery, University of Perugia, Perugia, Italy

${ }^{2}$ Department of Pharmaceutical Sciences, University of Perugia, Perugia, Italy

${ }^{3}$ Department of Health Sciences, University of Florence, Firenze, Italy

${ }^{4}$ Department of Medicine and Surgery, University of Parma,

Parma, Italy

${ }^{5}$ Department of Philosophy, Social Sciences and Education, University of Perugia, Perugia, Italy

Correspondence to

Dr Giorgia Renga;

rengagiorgia@gmail.com

\section{ABSTRACT}

Background Despite the great success, the therapeutic benefits of immune checkpoint inhibitors (ICls) in cancer immunotherapy are limited by either various resistance mechanisms or $\mathrm{ICl}$-associated toxic effects including gastrointestinal toxicity. Thus, novel therapeutic strategies that provide manageable side effects to existing ICls would enhance and expand their therapeutic efficacy and application. Due to its proven role in cancer development and immune regulation, gut microbiome has gained increasing expectation as a potential armamentarium to optimize immunotherapy with $\mathrm{ICl}$. However, much has to be learned to fully harness gut microbiome for clinical applicability. Here we have assessed whether microbial metabolites working at the interface between microbes and the host immune system may optimize ICI therapy.

Methods To this purpose, we have tested indole-3carboxaldehyde (3-IAId), a microbial tryptophan catabolite known to contribute to epithelial barrier function and immune homeostasis in the gut via the aryl hydrocarbon receptor (AhR), in different murine models of $\mathrm{ICl}$-induced colitis. Epithelial barrier integrity, inflammation and changes in gut microbiome composition and function were analyzed. AhR, indoleamine 2,3-dioxygenase 1, interleukin (IL)-10 and IL-22 knockout mice were used to investigate the mechanism of 3-IAld activity. The function of the microbiome changes induced by 3-IAld was evaluated on fecal microbiome transplantation (FMT). Finally, murine tumor models were used to assess the effect of 3-IAld treatment on the antitumor activity of ICI.

Results On administration to mice with $\mathrm{ICl}$-induced colitis, 3-IAld protected mice from intestinal damage via a dual action on both the host and the microbes. Indeed, paralleling the activation of the host AhR/IL-22-dependent pathway, 3-IAld also affected the composition and function of the microbiota such that FMT from 3-IAld-treated mice protected against ICl-induced colitis with the contribution of butyrate-producing bacteria. Importantly, while preventing intestinal damage, 3-IAld did not impair the antitumor activity of $\mathrm{ICl}$.

Conclusions This study provides a proof-of-concept demonstration that moving past bacterial phylogeny and focusing on bacterial metabolome may lead to a new class of discrete molecules, and that working at the interface between microbes and the host immune system may optimize ICl therapy.

\section{INTRODUCTION}

The recognition of the role of the immune system in cancer development has opened up novel therapeutic opportunities for cancer immunotherapy. Several strategies, including the use of chimeric antigen receptor $\mathrm{T}$ cells and immune checkpoint blockade, have represented major milestones in the therapy against cancer. Despite the great success of cancer immunotherapy using immune checkpoint inhibitors (ICIs), their therapeutic benefits are limited-60\%-70\% of patients do not respond to single agent immunotherapy-by either various resistance mechanisms ${ }^{1}$ or ICIassociated toxic effects, including frequent gastrointestinal, endocrine, and dermatological toxicities and fatal neurotoxicity and cardiotoxicity. ${ }^{2}$ Thus, novel therapeutic strategies that provide manageable side effects to existing ICI would enhance and expand their therapeutic efficacy and application, as recently suggested. ${ }^{3}$

Due to its proven role in cancer development and immune regulation, gut microbiome has gained increasing expectation as a potential armamentarium to improve cancer immunotherapy. ${ }^{45}$ Studies have shown that the effectiveness of immunotherapy against different tumors requires the presence of commensal bacteria. ${ }^{6-8}$ Clinical studies have corroborated these findings with compelling evidence that microbial richness and diversity are associated with a durable response to immunotherapy ${ }^{5}$ with gut microbiota signatures predicting toxicity associated with combined cytotoxic T-lymphocyte antigen 4 (CTLA-4) and programmed cell death protein 1 (PD-1) blockade. ${ }^{10}$ However, there 
is no identifiable pattern among the bacteria found to influence cancer therapies. Both Gram-positive and Gram-negative, both facultative and obligate anaerobes, and both coccus and rod-shaped bacteria are found to influence ICI treatment outcome and adverse effects (AEs).$^{11}$ Bacteroides, for instance, have shown a positive effect with anti-CTLA-4 therapies $^{8}$ but negative effects with anti-PD-1 therapies, being enriched in nonresponders ${ }^{9}$ and in patients with ICI-related toxicity. ${ }^{10}$ Thus, understanding how the repertoire of commensal microbes can be specifically manipulated to improve ICI therapeutic effectiveness has remained elusive. Considering that the gut microbiota potentially affects the efficacy of ICI through several mechanisms, including the production of critical metabolites, ${ }^{12}$ it is not surprising that the expression of certain metabolites is different in responders compared with non-responders to immunotherapy. ${ }^{13}$ This implicates that microbial metabolites can be used as predictive biomarkers of response to immunotherapy and points to a mean to optimize the efficacy of immune checkpoint blockade.

Microbiota-dependent tryptophan catabolites are abundantly produced within the gastrointestinal tract and are known to exert profound effects on host physiology, including the maintenance of epithelial barrier function and immune homeostasis. ${ }^{14}$ These metabolites are known to act as ligands of the aryl hydrocarbon receptor (AhR), a ligand-activated transcription factor first identified for its role in the binding and metabolism of xenobiotics and now implicated in a multiplicity of activities, such as the regulation of the immune response and the maintenance of the intestinal barrier homeostasis and microbial symbiosis. ${ }^{15}$ We have shown that indole-3-carboxaldehyde (3-IAld), produced in condition of unrestricted tryptophan availability in both mice ${ }^{1617}$ and humans, ${ }^{17-21}$ is a ligand of both murine and human $\mathrm{AhR}^{16}$ and evidence has since accumulated to link defective production of 3-IAld in certain clinical ${ }^{22-24}$ and preclinical ${ }^{25}$ settings involving impaired barrier function and mucosal homeostasis. $^{25} 26$

Based on these premises, in this study, we have evaluated whether and how 3-IAld could prevent intestinal toxicity, a common side effect of CTLA-4 inhibitors, ${ }^{2}$ while preserving the ICI therapeutic efficacy. To this purpose, we have administered an enteric formulation of 3-IAld, recently shown to increase its efficacy while minimizing unwanted toxicity through localized delivery in the small intestine, ${ }^{27}$ to mice with anti-CTLA-4-induced colitis. ${ }^{28}$ We found that 3-IAld protected mice from intestinal damage via a dual action on both the host and the microbe sides. Indeed, paralleling the activation of the host AhR/interleukin (IL)-22-dependent pathway, 3-IAld also affected the composition and function of the microbiota such that fecal microbiome transplantation (FMT) from 3-IAld-treated mice protected against ICI-induced colitis likely via butyrate-producing bacteria. Importantly, while preventing intestinal damage, 3-IAld did not impair the antitumor activity of anti-CTLA- 4 in a murine model of melanoma. Therefore, enteric formulated 3-IAld in combination therapy with ICI may preserve the mucosal integrity in the gastrointestinal tract without negatively affecting ICI therapeutic effectiveness.

\section{METHODS}

\section{Mice, models and treatments}

C57BL/6 mice were purchased from Charles River Labo-

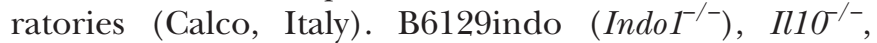

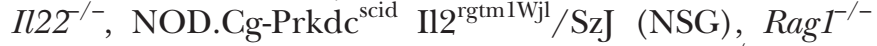
and B6.129-Ahrtm1Bra/J Ahr-deficient $\left(A h r^{--}\right)$mice were bred under specific pathogen-free conditions in the Animal Facility of Perugia (Perugia, Italy). The in vivo experiments performed in this work were approved by the Italian Ministry of Health. All knockout mice used in these studies were genotyped by PCR of DNA isolated from tail clippings. Male and female mice 5-10 weeks old were used in all experiments. Murine experiments were performed according to Italian Approved Animal Welfare Authorization 360/2015-PR and Legislative Decree 26/2014 regarding the animal license obtained by the Italian Ministry of Health lasting for 5 years (2015-2020). In the dextran sulfate sodium (DSS) colitis model, mice received $3 \%$ or $1 \%$ DSS (MP Biomedicals) in their drinking water for 7 days. Weight was recorded daily. Mice were injected intraperitoneally with $100 \mu \mathrm{g}$ of anti-CTLA-4 mAb (clone 9D9 BioXCell) or isotype control (clone MPC-11-BioXCell) two times (at days 0,4 , and 8 following the DSS administration). 6-Formylindolo(3,2-b) carbazole (FICZ) was administered intraperitoneally at the dose of $50 \mu \mathrm{g} / \mathrm{kg}$ for 5 days, beginning 2 days after the start of DSS administration. Butyrate $1 \%$ was administered in drinking water 1 week before the beginning of DSS administration. In the melanoma model, mice were subcutaneously injected into the right flank with $2 \times 10^{5}$ B16 tumor cells. Mice were injected four times at 3-day intervals with $100 \mu \mathrm{g}$ of isotype control or anti-CTLA-4 mAb. Tumor size, expressed in cubic millimeter, was measured by a caliper for 16 days following tumor inoculation. Tumor volume was determined every 2-3 days after inoculation (width ${ }^{2} \times$ length $\left./ 2\right) \times 1000$. In the Lewis lung carcinoma model, mice were intravenously injected with $2 \times 10^{5}$ LLC1 tumor cells. Mice were injected five times at 3-day intervals with $200 \mu \mathrm{g}$ of isotype control or anti-PD1 mAb (clone RMP1-14 BioXCell) and euthanized 18 days after tumor cell inoculation. In both DSS colitis and tumor models, 3-IAld was administered every other day at a dose of $18 \mathrm{mg} / \mathrm{kg}$, starting 4 days before DSS treatment or in concomitance with tumor challenge, as already described. ${ }^{28}$

\section{Models of immune-mediated colitis}

NSG mice 8-10 weeks old were injected intraperitoneally with $10^{7}$ human peripheral blood mononuclear cells (PBMCs). Human blood samples were obtained from healthy donors, and fresh PBMCs were isolated by density-gradient separation (Ficoll Paque Plus, GE 
Healthcare). Mice were treated intraperitoneally with $100 \mu \mathrm{g}$ of $\alpha \mathrm{hCTLA}-4$ or human IgG as antibody control (at days 4, 7, 10, 13 and 16). $\operatorname{Rag} 1^{-/-}$mice 8-10weeks old were injected intraperitoneally with $4 \times 10^{5} \mathrm{CD}^{+} \mathrm{T}$ cells and treated with anti-CTLA-4 mAb or control IgG the day after T-cell reconstitution and then on alternate days. ${ }^{29}$ $\mathrm{CD} 4^{+} \mathrm{T}$ cells were purified from spleens of $\mathrm{C} 57 \mathrm{BL} / 6$ naïve mice using anti-mouse CD4 (clone L3T4) coated MACS beads (Miltenyi Biotec) in accordance with the manufacturer's instructions. 3-IAld was administered every other day in concomitance with cells infusion. Mice were monitored daily for overall mortality and body weight loss and sacrificed on day 21.

\section{Enteric formulation preparation}

Briefly, the enteric microparticles (MPs) were prepared using Eudragit L100 to S100 (Rohm Pharma GmbH, Darmstadt, Germany) at a ratio of $1: 2$, with the addition EC $(30 \% \mathrm{w} / \mathrm{w}$, ETHOCEL std. 7; Dow Chemical Company, Milan, Italy), as described in Puccetti et al. ${ }^{30}$ 3-IAld (Sigma-Aldrich, Merck, Milan, Italy) and the polymers were dissolved in ethanol at a feedstock concentration of $3 \% \mathrm{w} / \mathrm{v}$ and spray-dried at an inlet temperature of $75^{\circ} \mathrm{C}$ using a Mini Spray-dryer model B-290 (Büchi, Milan, Italy) in the cocurrent mode, equipped with a two-fluid nozzle with a $0.7 \mathrm{~mm}$ nozzle tip and a $1.5 \mathrm{~mm}$ diameter nozzle cap. The aspirator capacity was maintained at $20 \mathrm{~m}^{3} /$ hour; the airflow rate was $301 \mathrm{~L} /$ hour; and the feed rate was $2.4 \mathrm{~mL} / \mathrm{min}$. The obtained dried MPs were recovered by using a high-performance cyclone (Büchi).

\section{Clinical signs and histopathology scores}

The severity of colitis was assessed by calculating disease activity index. All mice were monitored for stool consistency and rectal bleeding daily as described. ${ }^{28}$ Briefly, stool scores were determined as follows: 0, well-formed pellets; 1 , semiformed stools that did not adhere to the anus; 2, semiformed stools that adhered to the anus; and 3, liquid stools that adhered to the anus. Bleeding scores were determined as follows: 0 , no blood; 1 , positive hemoccult; 2, blood traces in stool visible; and 3, gross rectal bleeding. For histological evaluations, the tissues were removed and fixed in 10\% phosphate-buffered formalin (Bio Optica, Milan, Italy), embedded in paraffin, and sectioned at $3 \mu \mathrm{m}$. For histological analysis, sections were stained with periodic acid-Schiff (PAS) staining. The mucin production was evaluated with Alcian blue staining. For histological evaluations, colonic sections were examined and scored in a blinded fashion to assess four histological components: 'inflammation extent', 'damage in crypt architecture', 'hyperemia/edema', and 'grade of accumulation with inflammatory cells'. The colonic sections were scored from 0 to 3 points for each parameter. The total histological score, ranging from 0 to 12 , was obtained by summing the four histological component scores.

\section{Immunofluorescence and immunohistochemistry}

For immunofluorescence staining, sections were rehydrated and, after antigen retrieval in citrate buffer $(10 \mathrm{mM}, \mathrm{pH} 6)$, fixed in $2 \%$ formaldehyde for $40 \mathrm{~min}$ at room temperature and permeabilized in a blocking buffer containing 5\% fetal bovine serum (FBS), 3\% bovine serum albumin (BSA), and $0.5 \%$ Triton X-100 in PBS. The slides were then incubated at $4^{\circ} \mathrm{C}$ with primary antibodies anti-Ki67 (Abcam), anti-ZO-1 (Invitrogen), and anti-indoleamine 2,3-dioxygenase 1 (IDO1) (clone 10.1, Millipore). After extensive washing with PBS, the slides were then incubated at room temperature for $60 \mathrm{~min}$ with secondary antibodies, anti-mouse Alexa Fluor 555 (ThermoFisher Scientific) and anti-rabbit TRITC (Bethyl). Nuclei were counterstained with Hoechst 33342 (Invitrogen). For immunohistochemistry staining, sections were rehydrated and antigens were retrieved by boiling in a citrate buffer ( $10 \mathrm{mM}, \mathrm{pH}$ 6). Subsequently, the endogenous peroxidase was quenched with $3 \% \mathrm{H}_{2} \mathrm{O}_{2}$ for $10 \mathrm{~min}$ at room temperature and then incubated with a blocking buffer (10\% horse serum in tris-buffered saline (TBS). After rinsing, the slides were treated overnight at $4^{\circ} \mathrm{C}$ with primary antibodies anti-CD4 (clone GK1.5, Santa Cruz Biotechnology) and anti-CD8 (clone UCH-T4, Santa Cruz Biotechnology). The slides were then incubated with biotinylated anti-rat IgG or biotinylated anti-mouse IgG (Fisher Scientific and ThermoFisher Scientific). Antibody binding was detected with a VECTASTAIN Elite ABC Kit (Vector Laboratories, Maravai LifeSciences, Burlingame, California, USA). Diaminobenzidine was used as a chromogen, followed by counterstaining with hematoxylin. Images were acquired using a microscope BX51 and analySIS image processing software (Olympus). Infiltrated lymphocytes were observed at $\times 100$ magnification and expressed as mean of positive cell count around the tumor site per field (10 randomly selected high power fields/slide).

\section{Intestinal permeability}

Intestinal permeability was measured in fasted C57BL/ 6 mice for 4 hours prior to the administration of $40 \mathrm{mg} / 100 \mathrm{~g}$ mouse weight of fluorescein isothiocyanate (FITC)dextran (4 kDa, Sigma-Aldrich) as described.$^{28}$ Serum was collected retro-orbitally 4 hours later and diluted 1:3 in PBS. The amount of fluorescence at $488 \mathrm{~nm}$ for emission and absorption at $525 \mathrm{~nm}$ was read on the Infinite 200 plate reader (Tecan) using the manufacturer's I-control V.1.3 software.

\section{Kynurenine and tryptophan assay}

IDO1 functional activity was measured in colon homogenates in terms of the ability to metabolize tryptophan to kynurenine whose concentrations were measured by using competitive ELISA kits according to the manufacturer's instructions (Labor Diagnostika Nord).

ELISA and quantification of short-chain fatty acid (SCFA)

Murine IL-1 $\beta$, IL-10, IL-17A, IL-22 and tumour necrosis factor alpha (TNF- $\alpha)$ cytokine concentration as well as 
calprotectin levels were determined in colon homogenates. SCD14 was determined in serum by using specific ELISA kits according to the manufacturers' instructions (eBioscience, R\&D System and Biolegend). SCFAs in serum and feces were analyzed by gas chromatographymass spectrometry as described in online supplemental materials.

\section{Real-time PCR}

Real-time PCR was performed using the CFX96 Touch Real-Time PCR detection system and iTaq Universal SYBR Green Supermix (Bio-Rad). Organs were lysed and total RNA was extracted using TRizol Reagent (ThermoFisher Scientific) and reverse transcribed with PrimeScript RT Reagent Kit with gDNA Eraser (Takara) according to the manufacturer's directions. Amplification efficiencies were validated and normalized against $\beta$-actin. The murine primers used in this study are detailed in online supplemental materials.

\section{Detection of methylation in promoter of Foxp3 gene}

Total genomic DNA from mesenteric lymph nodes of mice was isolated using Nucleospin Tissue (Macherey-Nagel). Bisulfite modification of total DNA was performed using EpiTect Bisulfite Kit (Qiagen). To detect methylation/ demethylation in the promoter of Foxp3 containing CpG islands, real-time methylated PCR was performed using EpiTect MethyLight PCR (Qiagen). Bisulfite converted genomic DNA was amplified using mouse Foxp3-specific forward (5-GGGATTTAGGAGGGGATTT TTT-3) and reverse (5-GAAAAATTTTACCTAATACCCACATTTT-3) primer pairs and TaqMan hybridization probes (methylated Foxp3 probe: FAM-5-TCGTTTTAGGAGGCGG AGTAGCGTTTTT-3, demethylated Foxp3 probe: HEX-5TTGTTTTAGGAGGTGGTAGTAGTGTTTTT-3). Realtime methylated PCR reactions were carried out in a volume of $20 \mu \mathrm{L}$ using EpiTect MethyLight master Mix (Qiagen), 500 ng bisulfite converted genomic DNA, and pairs of forward and reverse primers $(0.4 \mu \mathrm{M})$ and TaqMan probes $(0.2 \mu \mathrm{M})$. Amplification and detection were carried out using the following profile: one step at $95^{\circ} \mathrm{C}$ for $5 \mathrm{~min}$, and 45 cycles at $95^{\circ} \mathrm{C}$ for $15 \mathrm{~s}$ and $58^{\circ} \mathrm{C}$ for $1 \mathrm{~min}$. The methylation index (\%) of each sample was calculated using the following equation: methylation index $=\mathrm{M} / \mathrm{M}+\mathrm{U} \times 100 \%$, where $\mathrm{M}$ is the quantity of methylated and $\mathrm{U}$ is the quantity of demethylated Foxp3 real-time MSP following bisulfite conversion, as already described. $^{31}$ All samples were run in duplicate and the average values were used.

\section{Sample collection, processing, and sequencing for microbial composition}

DNA was isolated from murine feces by the QIAamp Fast DNA Stool Mini Kit (Qiagen) according to the manufacturer's instructions. The bacterial microbiota was evaluated by $16 \mathrm{~S}$ rRNA sequencing performed at LGC Group (next-generation sequencing; Genomics, Berlin, Germany). The V4-V5 hypervariable regions of the bacterial $16 \mathrm{~S}$ rRNA gene were amplified by the 515YF/926R primer pair, and amplicons were sequenced on a 300 bp paired-end read sequencing on the Illumina MiSeq platform (V3). Statistical analysis was performed as described in online supplemental materials.

\section{Fecal microbiota transplantation}

Fresh fecal pellets from C57BL/ 6 control or 3-IAld-treated mice were collected, added with sterile PBS at $10 \mathrm{mg} / \mathrm{mL}$, homogenized, and centrifuged at $4000 \mathrm{rpm}$ for $10 \mathrm{~min}$ at $4^{\circ} \mathrm{C}$. The fecal preparations were administrated intragastrically to C57BL/6, $I l 1 \sigma^{--}$and $I l 22^{-/}$mice once a day 1 day before and 2 days after the induction of colitis. ${ }^{32}$

\section{Flow cytometry analysis}

For the Lewis lung carcinoma model, lungs containing orthotopic tumors were harvested, minced with scissors, and digested with Collagenase P (Sigma-Aldrich) and DNase in Hanks' balanced salt solution (HBSS) for $30 \mathrm{~min}$ at $37^{\circ} \mathrm{C}$. The total cell suspension was resuspended in FACS analysis buffer and then stained with the following antibodies for $30 \mathrm{~min}$ at $4^{\circ} \mathrm{C}$ in the dark: allophycocyanin (APC)-conjugated anti-CD4 (clone REA604, Miltenyi), APC Cy7-conjugated anti-CD45 (clone 104, BD Pharmingen), PE Cy7-conjugated anti-B220 (clone RA-6B2, BioLegend), SB600-conjugated anti-CD3 (clone 145-2C11, ThermoFisher Scientific), PE Cy7-conjugated anti-CD25 (clone PC61, BD Pharmingen), AF488-conjugated anti-Foxp3 (clone 150D, BioLegend). Intracellular staining was conducted using the Cytofix/ Cytoperm plus kit (BD PharMinigen). After staining, the cells were washed with FACS PBS and quantified using the BD LSRFortessa cell analyzer (Becton Dickinson). Gating strategy has been shown in online supplemental figure S1.

\section{Statistical analysis}

Student's t test, one-way and two-way analysis of variance with Bonferroni post hoc test was used to determine the statistical significance. Significance was defined as $p<0.05$. Data are pooled results $($ mean $\pm \mathrm{SD}$, mean $\pm \mathrm{SEM})$ or representative images from two or three (for the murine colitis model) or two experiments (for the tumor models). The histological scores were compared using the KruskalWallis test. The in vivo groups consisted of three to six mice/group. Mice were allocated in each group by simple randomization. No criteria were set for including/ excluding animals during the experiments. The order of treatment was maintained throughout the experiment. No blinding procedures were used. No animals were excluded in the analysis. Sample size was decided based on our previous manuscript with the same experimental models. ${ }^{28}$ GraphPad Prism software V.6.01 (GraphPad Software) was used for analysis.

\section{RESULTS}

\section{3-IAld protects against ICI-induced colitis}

To evaluate whether enteric formulated 3-IAld would rescue from intestinal damage and toxicity in ICI-induced colitis, we resorted to a murine model of ICI-induced 
colitis in which the vigorous T-cell response promoted by anti-CTLA- 4 treatment exacerbated the DSS-induced intestinal damage. ${ }^{33} 34$ Administration of 3-IAld starting the week before and continuously until the end of the experiment (figure 1A) improved survival (figure 1B), prevented weight loss (figure 1C), ameliorated disease activity index (figure 1D), and improved gross pathology (figure 1E) in colitic mice. Of note, the protective effects of 3-IAld were comparable with those observed on the administration of the high-affinity AhR ligand, FICZ (figure 1B-E). Similar results were obtained in models of immune-mediated colitis obtained by infusion of human PBMCs or murine $\mathrm{CD} 4^{+} \mathrm{T}$ cells in NSG (figure $1 \mathrm{~F}-\mathrm{J}$ ) or RAG1-deficient mice (online supplemental figure S2), respectively, and subsequent treatment with anti-CTLA-4. The treatment with 3-IAld increased survival (figure $1 \mathrm{~F}$ ), reduced weight loss (figure 1G), improved inflammatory histopathology and barrier function, as revealed by the expression of the tight junction zonula occludens 1 (ZO-1) (figure 1H,I), and decreased inflammatory cytokine production (figure 1J) in NSG and RAG1-deficient mice (online supplemental figure S2). Similarly, the protective effects of 3-IAld were associated with an improved colon histopathology (figure 2A,B) and intestinal barrier integrity in mice with DSS-colitis, as revealed by ZO-1 expression and proliferation of intestinal stem cells, revealed by $\mathrm{Ki}-67$ staining (figure $2 \mathrm{C}$ ) and $\mathrm{Lrg} 5$ expression (figure 2D). Of interest, 3-IAld also promoted the expression of $N f i l 3$, a transcription factor that directs the development of innate lymphoid cells (ILC) ${ }^{35}$ known to maintain epithelia barrier integrity ${ }^{36}$ as well as of Muc1, a cell surface mucin that functions as a barrier to infection and as regulator of inflammation ${ }^{37}$ (figure 2D). In agreement with these data, the passage of dextran-FITC across the intestinal barrier was reduced (figure 2E), and the levels of soluble CD14, a marker of gut permeability, ${ }^{38}$ were decreased (figure $2 \mathrm{~F}$ ). These changes were paralleled by a switch towards an anti-inflammatory profile with reduced levels of TNF- $\alpha$, IL-1 $\beta$ and IL-17A, and increased amounts of IL-10 (figure 2G). Consistent with the antiinflammatory profile, the levels of calprotectin were also reduced (figure 2H). Considering that 3-IAld is defective in mice with colitis, ${ }^{25}$ these results indicate that 3-IAld supplementation may protect against ICI-induced colitis by maintaining epithelial barrier integrity and dampening the inflammatory response, an activity consistent with its AhR-agonistic activity and comparable to FICZ.

\section{Beneficial effects of 3-IAId are not dependent on ID01 and IL-10}

We have previously demonstrated that IDO1, an enzyme involved in the kynurenine pathway of tryptophan metabolism, ${ }^{39}$ mediated protection from intestinal pathology in a murine model of ICI-induced colitis with increased production of IL-10. ${ }^{28}$ We evaluated whether the IDO1/ IL-10 pathway was involved in the therapeutic activity of 3-IAld. As shown in figure 3, 3-IAld increased neither IDO1 gene or protein expression (figure 3A,B) nor its enzymatic activity (figure 3C), as measured by the kynurenine/tryptophan ratio. Accordingly, the downstream genes Haao and Kynu were not increased on administration of 3-IAld (figure 3D), likely indicating that the activity of 3-IAld occurs independently of IDO1 engagement. Experiments in IDO1-deficient mice with ICI-colitis confirmed that the protective effects of 3-IAld did not involve IDO1 (figure 3E,F). Likewise, experiments in IL-10-deficient mice revealed that the activity of 3-IAld did not involve IL-10 either. Due to the existing epithelial damage, a chronic enterocolitis develops in these mice by 2-3 months of age. ${ }^{40}$ Therefore, we administered 3-IAld, as indicated in figure 1A, to 10-week-old mice treated with a lower dosage of DSS with and without concomitant antiCTLA-antibody. We first noticed that IL-10-deficient mice at variance with wild-type mice developed signs of colitis on anti-CTLA-4 treatment alone (figure 3G), one first finding demonstrating that anti-CTLA4 treatment alone can drive intestinal inflammation de novo. As expected, the severity of colitis increased on concomitant DSS and anti-CTLA-4 treatment (figure 3I,J). Strikingly, 3-IAld not only promoted mucin homeostasis as seen on PAS/Alcian blue staining and the resolution of the rectal prolapse (online supplemental figure S3) in IL-10-deficient mice with DSS-colitis but also prevented weight loss (figure 3H) and improved histopathology (figure $3 \mathrm{G}, \mathrm{I}, \mathrm{J}$ ) in these mice on anti-CTLA- 4 treatment alone or combined with DSS. These results would indicate that 3-IAld protects from epithelial damage and immune-mediated damage in the relative absence of IL-10.

\section{Beneficial effects of 3-IAld involve the AhR/IL-22 axis}

It has been suggested that signaling by indoles in response to inflammation induced by acute stressors occurs through IL-22, ${ }^{41}$ a cytokine that depends on $\mathrm{AhR}^{42}$ and partners with IL-10 in maintaining mucosal homeostasis. ${ }^{43}$ We found that administration of 3-IAld to DSS +anti-CTLA-4 antibody-treated mice induced the expression of the AhR-dependent genes Cypla1 and Ahrr (figure 4A), a finding suggesting AhR engagement. Experiments in AhR-deficient mice confirmed the lack of 3-IAld's activity in these mice (figure 4B,C) as already described. ${ }^{16}$ Moreover, 3-IAld also induced IL-22 gene and protein expression (figure 4A,D), as well as the downstream target $R e g 3 \gamma$ (figure 4E), an antimicrobial peptide produced by intestinal epithelial cells. In agreement with previous findings ${ }^{16}$ experiments in IL-22-deficient mice directly proved that IL-22 is instrumental for 3-IAld's activity in colitis. We found that the weight loss (figure 4F), disease activity index (figure 4G), and colon histopathology (figure 4H,I) were not rescued by 3-IAld in these mice. Similarly, 3-IAld neither prevented epithelial barrier damage, as shown by ZO-1 staining (figure 4I), nor reverted the inflammatory profile (figure $4 \mathrm{~J}$ ) in the relative absence of IL-22. These results indicate that the protective activity of 3-IAld against colitis depends on AhR and IL-22, while IDO1 and IL-10 are dispensable. 
A

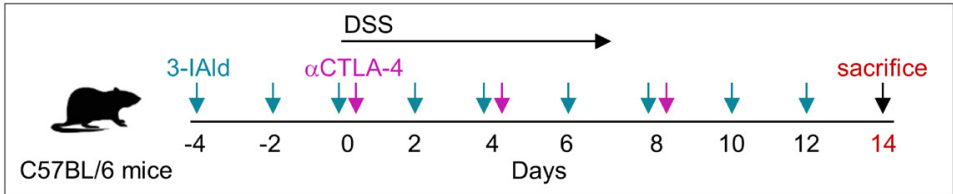

B

C
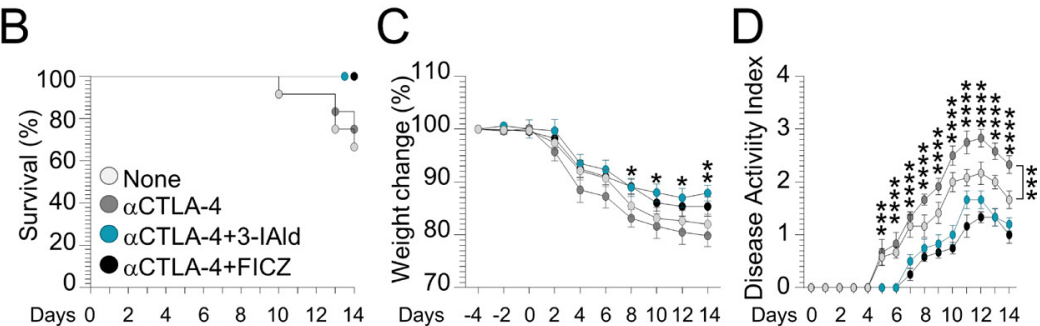

E

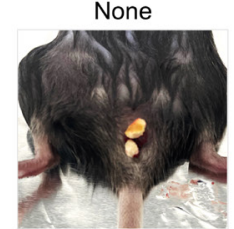

$\alpha$ CTLA-4

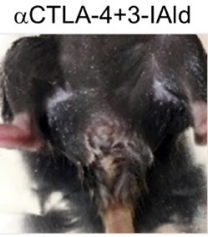

$\alpha$ CTLA-4+FICZ

F
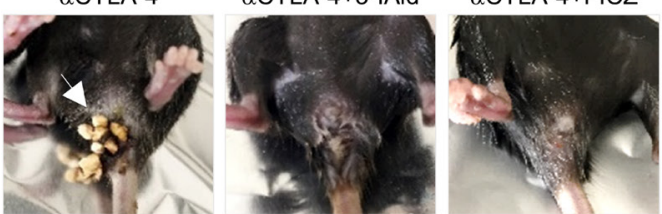

G

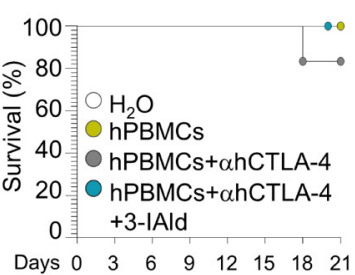

$\mathrm{H}$
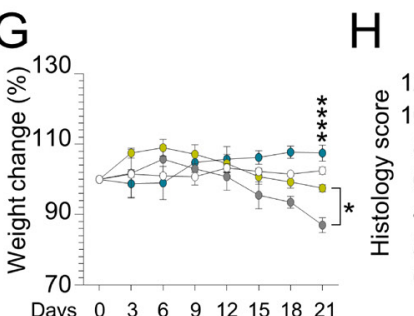

I

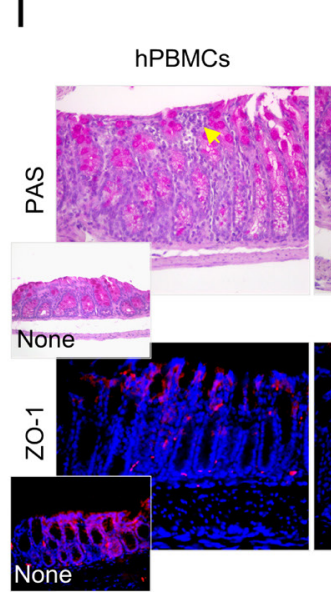

hPBMCs+ ahCTLA-4

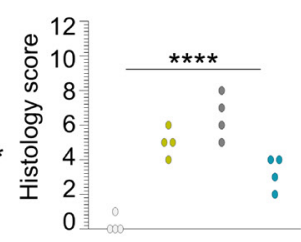

$\mathrm{J}$
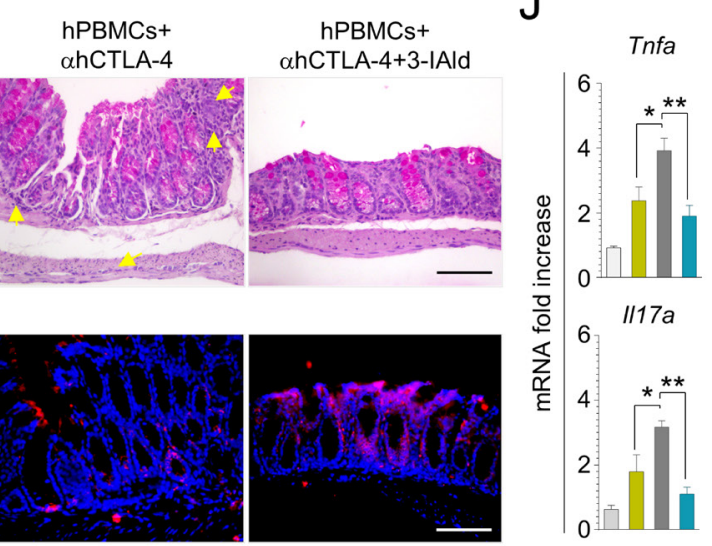

Figure 1 3-IAld protects mice from ICl-induced colitis. C57BL/6 mice were treated with DSS in drinking water for 1 week followed by a recovery period of another week, and administered $100 \mu \mathrm{g}$ of anti-CTLA-4 mAb or isotype control two times (at days 0,4 , and 8 following DSS administration). 3-IAld $(18 \mathrm{mg} / \mathrm{kg})$ was administered intragastrically every other day starting 4 days before DSS treatment. FICZ was used as control as depicted in the experimental schedule (A). Mice were evaluated for (B) \% survival, (C) \% weight change, (D) disease activity index, and (E) rectal bleeding. NSG mice infused intraperitoneally with hPBMCs were treated with ohCTLA-4 and 3-IAld. Mice were sacrificed at 21 days and evaluated for (F) \% survival, (G) \% weight change, (H) histology score, (I) colon histology (PAS staining) and ZO-1 protein expression and (J) inflammatory cytokine expression. Photographs were taken with a high-resolution microscope (Olympus BX51), ×20 magnification (scale bars, $200 \mu \mathrm{m})$. White arrow indicates rectal bleeding. Yellow arrows indicate inflammatory cells recruitment. For histology, data are representative of two or three independent experiments. Each in vivo experiment includes four to six mice per group (16-24 mice in each experiment). Data are represented as mean \pm SEM. (B-D) Anti-CTLA-4-treated mice versus none (isotype control), anti-CTLA-4+3-IAld- versus anti-CTLA-4-treated mice, (F-J) hPBMCs+ $\alpha$ hCTLA-4 versus hPBMCs (+human lgG) or hPBMCs+ $\alpha$ hCTLA-4+3 IAld. Two-way analysis of variance, Bonferroni post hoc test. $\mathrm{H}_{2} \mathrm{O}$, untreated mice. ${ }^{\star} \mathrm{P}<0.05$, ${ }^{\star *} \mathrm{P}<0.01$, ${ }^{* \star *} \mathrm{P}<0.001,{ }^{* * *} \mathrm{P}<0.0001$. 3-IAld, indole-3-carboxaldehyde; FICZ, 6-formylindolo(3,2-b)carbazole; hPBMC, human peripheral blood mononuclear cell; $\mathrm{ICI}$, immune checkpoint inhibitor; PAS, periodic acid-Schiff. 
A

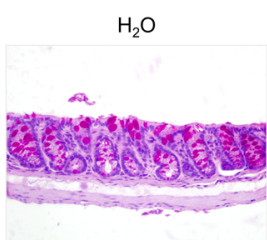

C

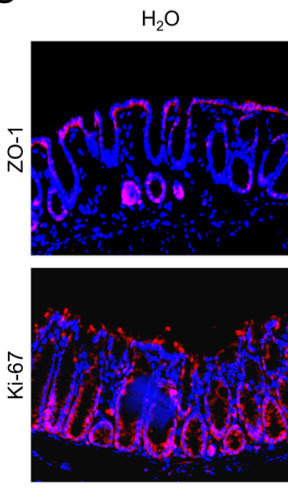

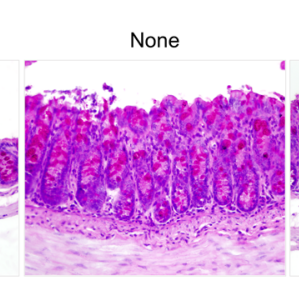
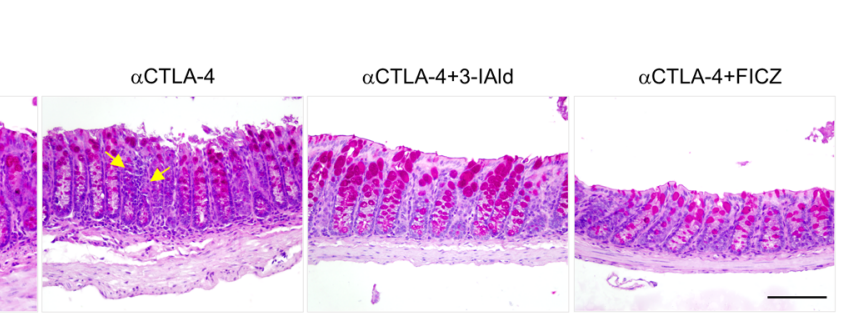

B

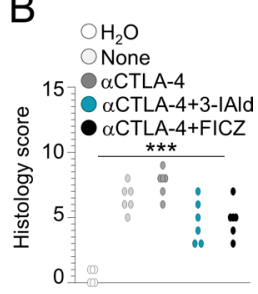

$\mathrm{E}$
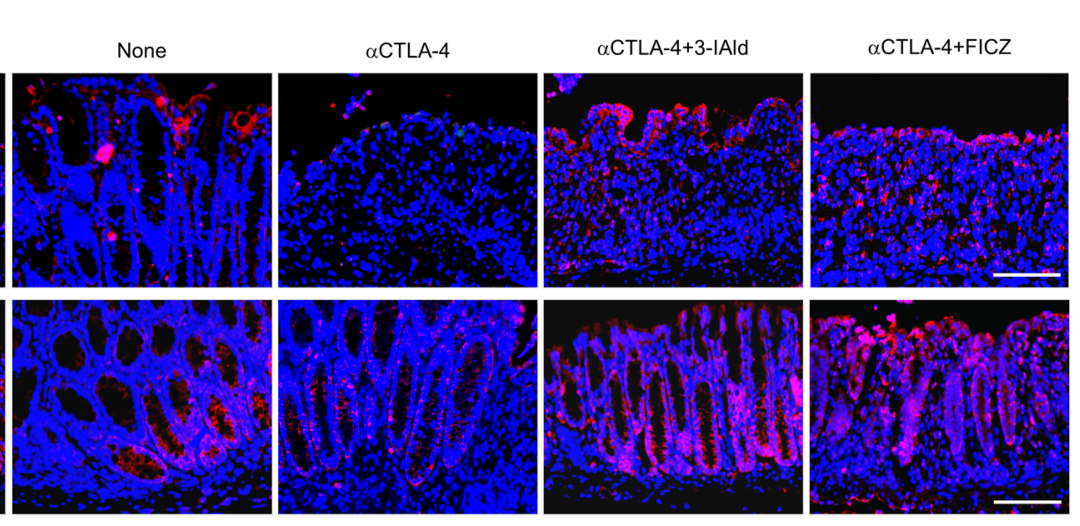

G

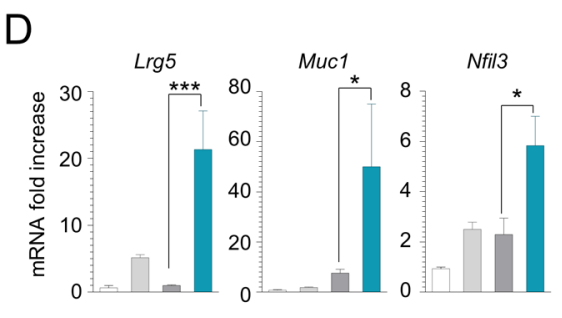

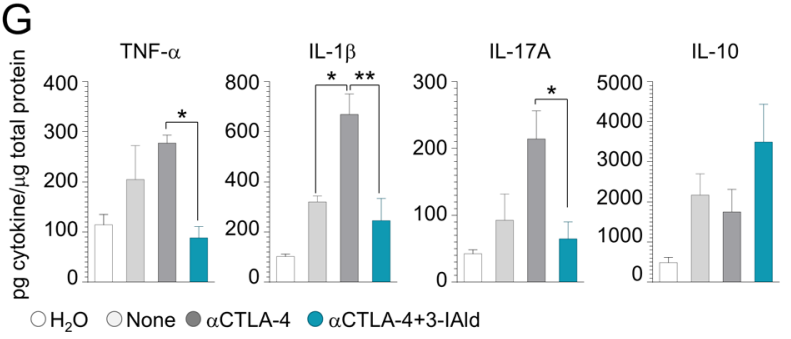

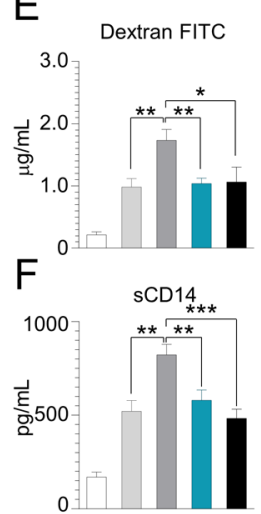

$\mathrm{H}$

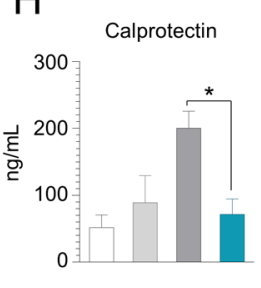

Figure 2 3-IAld prevents epithelial damage in DSS plus anti-CTLA-4-induced colitis. C57BL/6 mice were subjected to DSS plus anti-CTLA-4-induced colitis and administered 3-IAld as described in the legend of figure 1. Mice were evaluated for (A) colon histology (periodic acid-Schiff staining), (B) histology score, (C) ZO-1 and Ki-67 protein expression, (D) intestinal stem cells genes expression, (E) Dextran-FITC and (F) sCD14 levels in the serum, $(G)$ cytokine and $(H)$ calprotectin levels in colon homogenates. For immunofluorescence, nuclei were counterstained with Hoechst 33342. Photographs were taken with a high-resolution microscope (Olympus BX51), $\times 40$ magnification (scale bars, $100 \mu \mathrm{m})$. Yellow arrows indicate inflammatory cell recruitment. For histology and immunofluorescence, data are representative of three independent experiments. Each in vivo experiment includes three to six mice per group (15-30 mice in each experiment). Data are represented as mean \pm SEM, (B) Kruskal-Wallis test. (D-H) Anti-CTLA-4- versus none (isotype control) or anti-CTLA-4+3-IAld-treated mice. One-way analysis of variance, Bonferroni post hoc test. $\mathrm{H}_{2} \mathrm{O}$, untreated mice. ${ }^{*} \mathrm{P}<0.05$, ${ }^{\star \star} \mathrm{P}<0.01$, ${ }^{* \star \star} \mathrm{P}<0.001$. 3-IAld, indole-3-carboxaldehyde; FICZ, 6-formylindolo(3,2-b)carbazole; IL, interleukin; TNF- $\alpha$, tumour necrosis factor alpha.

\section{3-IAld modifies intestinal microbiota composition and function}

In addition to preserving an intact epithelium, the AhR/IL-22 axis maintains a balanced microbiota and a functioning defense system. ${ }^{44}$ We therefore evaluated whether treatment with 3-IAld would affect the fecal microbial composition. Bacteroidota represented the most abundant phyla in murine feces followed by Firmicutes, while Proteobacteria and Actinobacteria were present at very low abundances (figure 5A). At genus level, Muribaculaceae (former S24-7) were the most abundant, followed by members of Clostridia (Lachnospiraceae and Oscillospiraceae) and Bacteroidia (Prevotellaceae, Rikenellaceae, and Bacteroidaceae) (online supplemental figure S4). The analysis of alpha diversity did not reveal significant differences in richness and evenness between untreated and 3-IAld-treated groups, as measured by observed operational taxonomic units (OTUs), Chaol and Shannon indexes (figure 5B). On the contrary, significant differences were observed in compositional structure, as measured by Jaccard and Bray-Curtis indexes (figure 5C). Indeed, PCoA analysis derived from Jaccard and Bray-Curtis distances revealed the presence of distinct clusters (online supplemental figure S5). In order to identify putative 3-IAld microbial signatures, we performed high-dimensional class comparisons using LEfSe. As shown in figure 5D,E, we observed that treatment with 3-IAld was associated with a predominance of members of Bacteroidota (Prevotellaceae, Bacteroidaceae, and Muribaculaceae), Desulfobacterota and, among Firmicutes, of Lachnospiraceae (Roseburia genus). Increased levels of alphaproteobacteria and different members of Bacteroidota (Rikenellaceae and Bacteroidales F082) were instead observed 
A

B
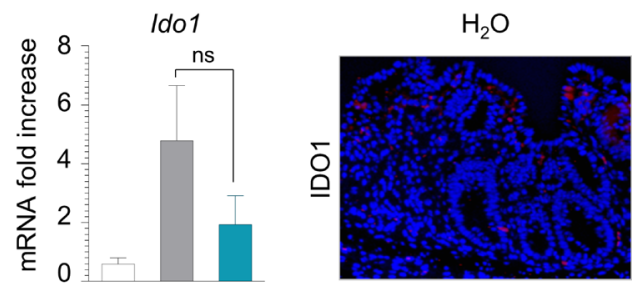

DSS $+\alpha C T L A-4$

DSS $+\alpha$ CTLA-4+3-IAld

C

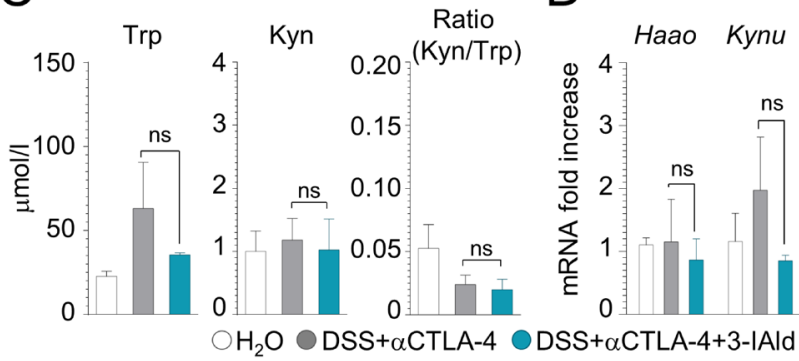

E
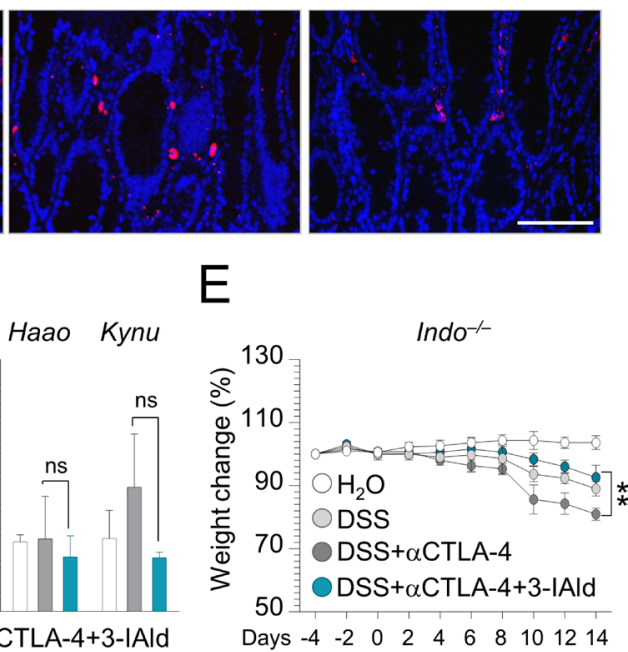

F

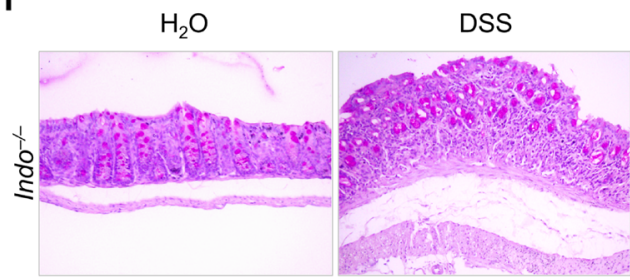

G
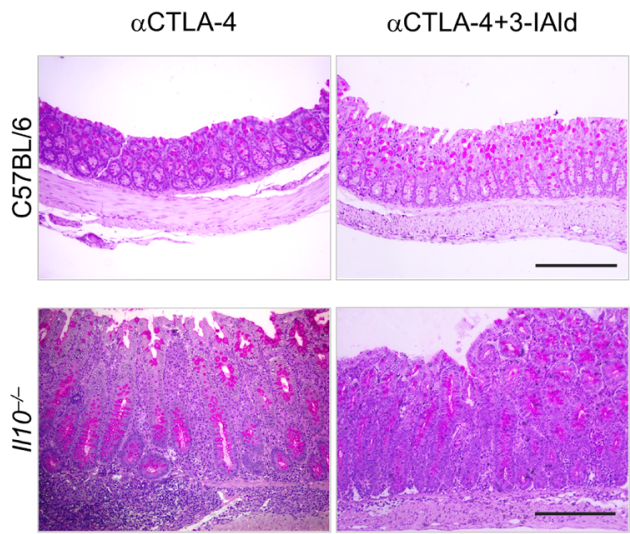

J

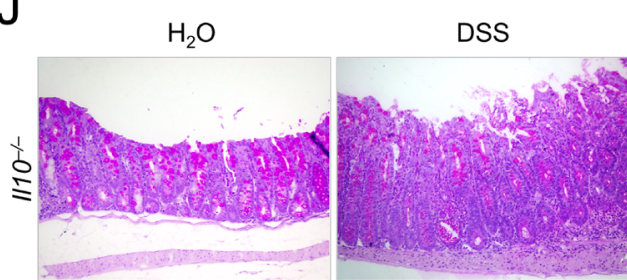

DSS $+\alpha$ CTLA -4

DSS $+\alpha$ CTLA-4+3-IAId

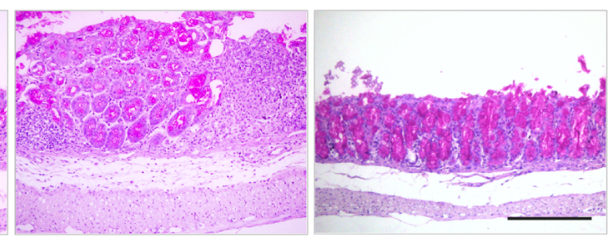

$\mathrm{H}$

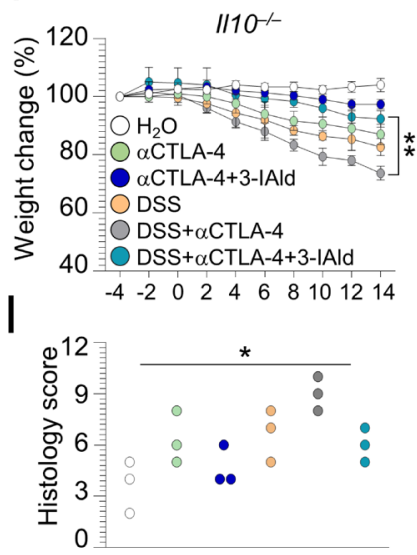

DSS $+\alpha$ CTLA- 4 DSS $+\alpha$ CTLA-4+3-IAId
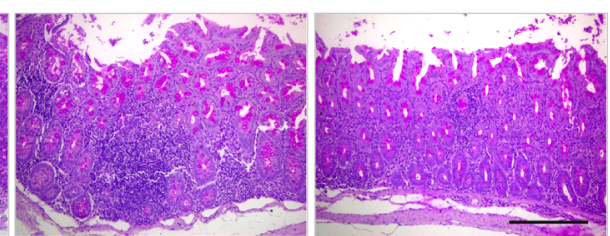

Figure 3 Protective activity of 3-IAld is not dependent on IDO1 and IL-10. C57BL/6 (A-D,G), Indo $1^{-/-}(\mathrm{E}-\mathrm{F})$ and $/ / 10^{-/-}$ (G-J) mice were subjected to anti-CTLA-4 with (A-F,H-J) or without (G) DSS and administered 3-IAld as described in the legend of figure 1. Mice were evaluated for (A) IDO1 gene and (B) protein expression, (C) Trp, Kyn levels and Kyn:Trp ratio, (D) Haao and Kynu expression, (E,H) \% weight change, (F-J) colon histology (periodic acid-Schiff staining), (I) histology score. For immunofluorescence, nuclei were counterstained with Hoechst 33342. Photographs were taken with a highresolution microscope (Olympus BX51), ×20 magnification (scale bar, $200 \mu \mathrm{m}$ ). For histology and immunofluorescence, data are representative of three independent experiments. Each in vivo experiment includes 3 mice per group (9-18 mice total). Data are represented as mean \pm SEM. Anti-CTLA-4+3-IAld- versus anti-CTLA-4-treated mice. One-way analysis of variance, Bonferroni post hoc test, (I) Kruskal-Wallis test. $\mathrm{H}_{2} \mathrm{O}$, untreated mice. ${ }^{*} \mathrm{P}<0.05$, ${ }^{* *} \mathrm{P}<0.01$. 3-IAld, indole-3-carboxaldehyde; IDO1, indoleamine 2,3-dioxygenase 1; IL, interleukin; Kyn, kynurenine; ns, not significant; Trp, tryptophan. 

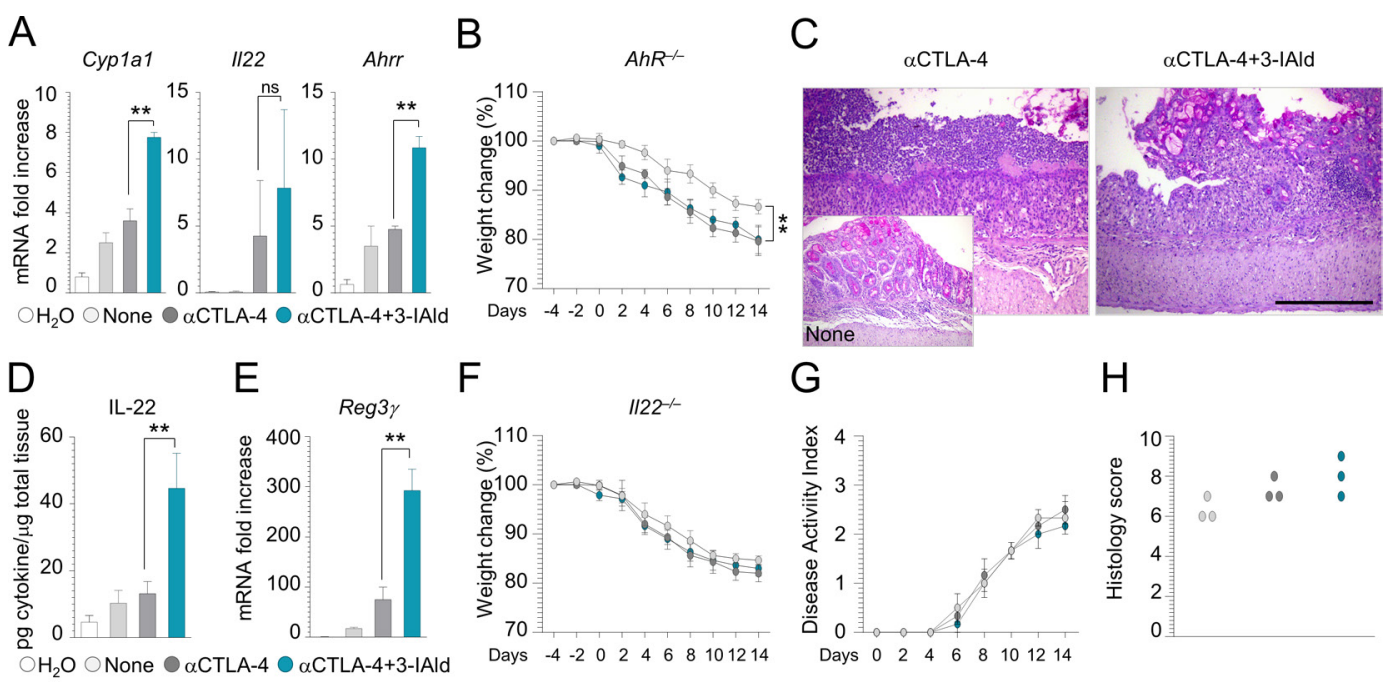

I
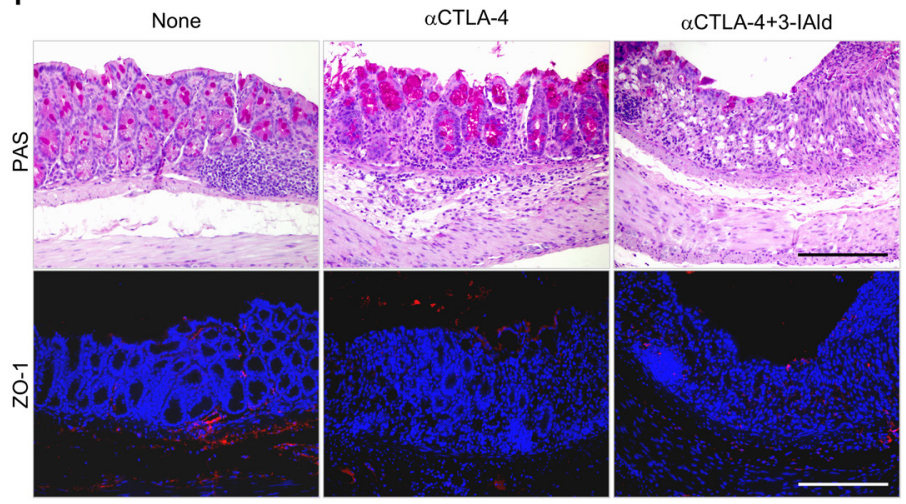

$\mathrm{J}$

Figure 4 Beneficial effect of 3-IAld involves the AhR/IL-22 axis. C57BL/6 (A,D,E), AhR ${ }^{-/}$(B,C), II22 ${ }^{-/-}$(F-J) mice were subjected to DSS plus anti-CTLA-4-induced colitis and administered 3-IAld as described in the legend of figure 1. Mice were evaluated for (A) AhR-related genes, (B,F) \% weight change, (C,I) colon histology (periodic acid-Schiff staining), (D) IL-22 levels, (E) Reg3y gene expression, (G) disease activity index, (H) histology score, (I) ZO-1 expression, and (J) cytokine production in colon homogenates. For immunofluorescence, nuclei were counterstained with Hoechst 33342. Photographs were taken with a highresolution microscope (Olympus BX51), ×20 magnification (scale bars, $200 \mu \mathrm{m}$ ). For histology and immunofluorescence, data are representative of three independent experiments. Each in vivo experiment includes 3 mice per group (9-12 mice in each experiment). Data are represented as mean \pm SEM. Anti-CTLA-4+3-IAld- versus anti-CTLA-4-treated mice. One-way analysis of variance, Bonferroni post hoc test, $(F)$ Kruskal-Wallis test. $\mathrm{H}_{2} \mathrm{O}$, untreated mice. None, DSS+isotype control. ${ }^{* *} \mathrm{P}<0.01$. 3-IAld, indole-3-carboxaldehyde; IL, interleukin; ns, not significant; TNF- $\alpha$, tumour necrosis factor alpha.

in untreated mice. We then resorted to Phylogenetic Investigation of Communities by Reconstruction of Unobserved States 2 (PICRUSt2) and the KEGG database to determine the abundance of functional modules inferred from 16S data, and LEfSe was applied in order to evaluate significant association in either untreated or 3-IAld-treated samples. As shown in figure 5F, pectin degradation followed by transport systems, such as phosphate, lipopolysaccharide, tungstate, glutamine and the phosphoenolpyruvate-carbohydrate phosphotransferase (PTS) system, and sulfur reduction, were the modules most increased by 3-IAld, while biosynthetic pathways, that is, ubiquinone, fatty acids, polyamine, cysteine and threonine biosynthesis, were reduced. The relative abundance of pectin-degrading and sugar-degrading anaerobic bacteria on 3-IAld treatment led us to assess the fecal and serum levels of SCFAs that, together with different gases (including hydrogen sulfide, $\mathrm{H}_{2} \mathrm{~S}$ ), are known end products of bacterial fermentation of pectin. ${ }^{46}$ Consistent with the selective increase of the genus Roseburia, known to produce high levels of butyrate, ${ }^{47}$ the fecal levels of n-butyric acid were significantly increased along with those of valeric acid and, to a lesser extent, propionic acid (figure 5G). However, SCFAs were not increased, if not decreased for the acetic acid, at the systemic level (figure 5G). Thus, the combined metagenomics and metaproteomic analysis revealed that 3-IAld is able to shift the microbial composition toward sugar-fermenting bacteria and localized SCFA production.

\section{3-IAld-modified microbiota provides protection to ICI-induced colitis}

We resorted to fecal microbiota transplantation to evaluate the activity of 3-IAld-modified microbiota in colitis. Feces were collected from untreated or 3-IAld-treated mice for 6 days and transplanted (FMT) into recipient 
A

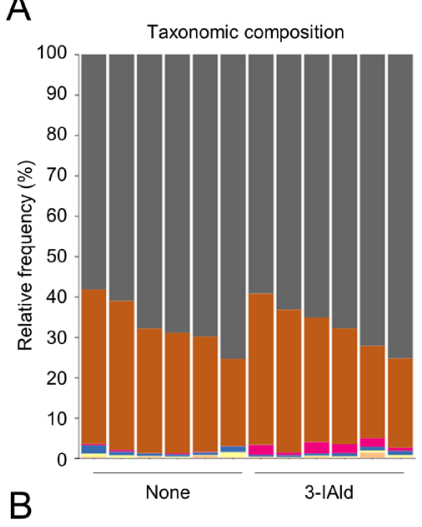

B
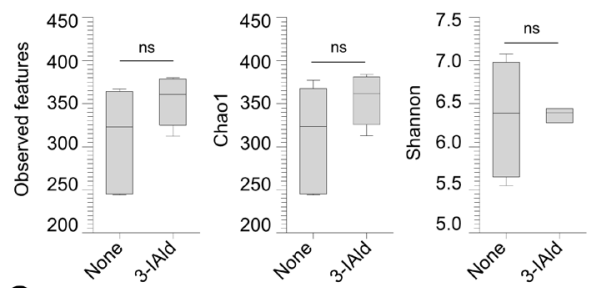

C
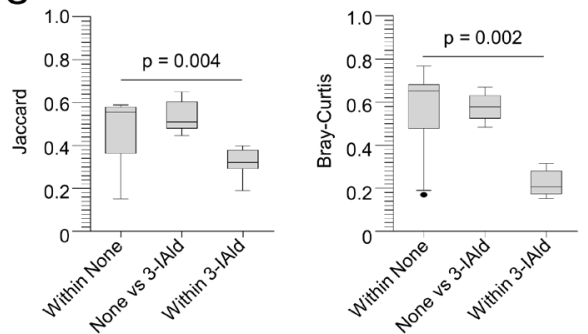

G

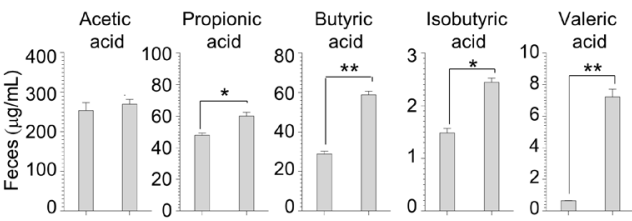

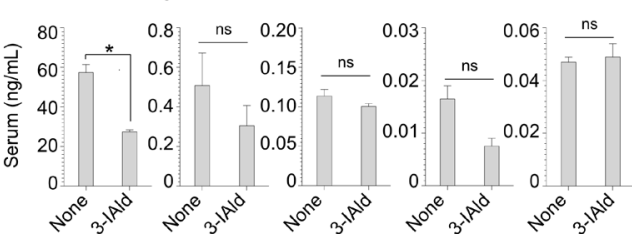

D

$\mathrm{E}$
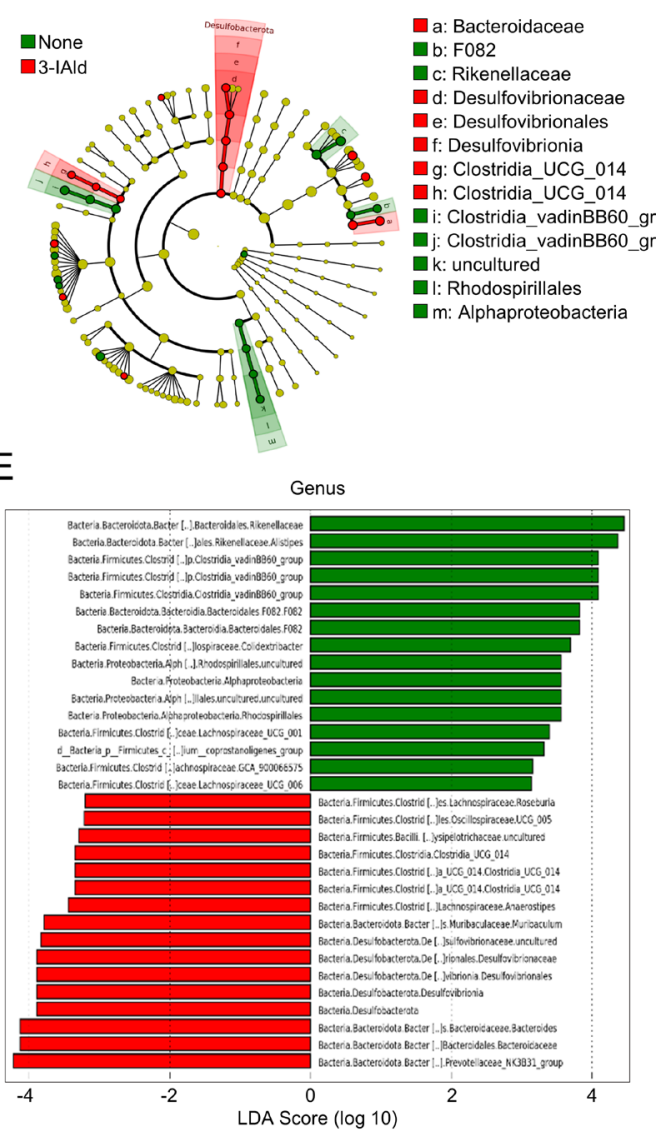

$\mathrm{F}$

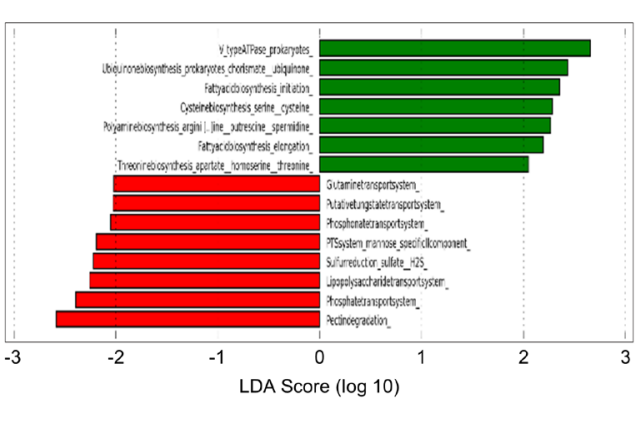

Figure 5 3-IAld modifies intestinal microbiota composition and function. (A) Barplot showing bacterial composition (abundance percentage) of each sample at phylum level. Taxa are differentiated by colors. Samples are ranked based on the abundance of the most abundant Phylum (Bacteroidota) and grouped by None and 3-IAld-treated. (B) Boxplots of observed features, Chao1 and Shannon alpha diversity indexes. Significance was evaluated by applying a Kruskal-Wallis test (ns).

(C) Boxplots of Jaccard and Bray-Curtis beta diversity indexes evaluating distances within or between none and 3-IAld-treated samples. Significance was evaluated by applying a Kruskal-Wallis test (the $p$ value is indicated). (D,E) LEfSe at genus level. LEfSe emphasizes a set of features that significantly discriminate between none and 3-IAld treatments. A p value of $<0.05$ and an LDA score of $\geq 3.5$ were regarded as significant in Kruskall-Wallis and pairwise Wilcoxon tests, respectively. The cladogram (D) simultaneously highlights both phyla and specific genera. Taxa (circles) are colored green when significantly associated to none, red when significantly associated with 3-IAld-treated samples, and yellow when not significantly associated to either groups. The size of each circle is proportional to the abundance of the corresponding taxon in all samples. The barplot of the LDA scores (E) shows genera significantly associated with either none and 3-IAld-treated samples accordingly to legend colors. LEfSe has been applied with default alpha values. (F) LEfSe on KEGG modules. Barplot of the LDA score computed on metabolic functions inferred by PICRUSt2 analysis (KEGG modules) and significantly associated with either none or 3-IAldtreated samples accordingly to legend colors. The threshold value of the logarithmic LDA score was set to 2.0. (G) Levels of SCFA measured in the feces and serum by mass spectrometry. (A-F) Six mice per group (12 mice total); (G) 3-6 mice per group (9 mice total). Data are represented as mean $\pm S D$. None versus 3 -IAld, $t$ test. ${ }^{*} P<0.05,{ }^{* *} P<0.01$. 3-IAld, indole-3carboxaldehyde; LDA, linear discriminant analysis; LEfSe, linear discriminant analysis together with effect size evaluation; ns; not significant. 
mice before and at the onset of colitis. ${ }^{32}$ At variance with transfer of feces from untreated mice, the transplantation of feces from 3-IAld-treated mice prevented weight loss (figure 6A), and ameliorated colon histopathology (figure 6B-D) in DSS-treated mice and similarly in DSS +anti-CTLA-4-treated mice (figure 6E). The reduced colonic inflammation was accompanied by the induction of IL-10-producing regulatory T cells (Treg) (figure 6F), as revealed by the reversal of DNA hypermethylation of Foxp3 promoter, likely occurring via butyrate (figure 6F), as already shown. ${ }^{44}$ Indeed, the finding that the protective activity of feces from 3-IAld-treated mice involved host IL-22 more than IL-10 (figure 6G-I), indicates that the beneficial activity of 3-IAld may occur through different pathways that include the modification of the composition and function of the microbiota, the increase intestinal barrier via the AhR/IL-22 axis and the control of inflammation via Treg cells.

\section{3-IAld does not interfere with the antitumor activity of anti- CTLA-4 antibody}

The potential application of 3-IAld requires that the protection against side effects does not compromise the therapeutic efficacy of ICI. For this reason, we assessed the effect of 3-IAld in the anti-CTLA-4-responsive B16 melanoma model. 3-IAld neither modified tumor growth nor interfered with the therapeutic efficacy of anti-CTLA-4 antibody (figure $7 \mathrm{~A}, \mathrm{~B}$ ) and did not affect recruitment of $\mathrm{CD} 4^{+}$and $\mathrm{CD}^{+}$tumor-infiltrating lymphocytes (figure 7C,D), consistent with the increased expression of leukocyte-recruiting chemokine Cxcl9 and effector Perforin (figure 7E). Likewise, 3-IAld did not interfere with the therapeutic efficacy of anti-PD-1 antibody in a model of Lewis lung carcinoma. Indeed, 3-IAld did not prevent the ability of anti-PD-1 antibody to increase survival (figure 7F), decrease tumor growth (figure 7G), improve gross pathology (figure $7 \mathrm{H}$ ), and reduce the recruitment of Foxp $3^{+} \mathrm{CD} 25^{+}$Treg cells in the lung (figure $7 \mathrm{I}, \mathrm{J}$ ). Thus, the beneficial activity of 3-IAld may extend to combination immunotherapy.

\section{DISCUSSION}

This study is a proof-of-concept demonstration of the therapeutic potential of bacterial metabolites as biologics capable of alleviating ICI-induced intestinal toxicity without interfering with ICI's antitumor activity. We found that 3-IAld, directly delivered in the intestine, activated the AhR/IL-22 pathway for epithelial barrier function and immune homeostasis during colitis. Despite the ability of 3-IAld to promote IL-10R1 expression in the gut ${ }^{25}$ and the ability of the IDO1/ IL-10 pathway to afford protection from ICI-induced intestinal damage, ${ }^{28}$ we did not find the involvement of this pathway in our setting. Interestingly, however, the chronic administration of 3-IAld (online supplemental figure S6A) only slightly prevented weight loss (online supplemental figure S6B) and improved colon histopathology in DSS colitis of aged IL-10-deficient mice (online supplemental figure S6C,D), implicating a possible role for IL-10 in mediating the clinical response of 3-IAld during chronic intestinal epithelial damage.

Gastrointestinal inflammation is a common immunerelated AE of anti-CTLA-4 treatment due to alterations in gut barrier integrity. ${ }^{48}$ However, decreased gut barrier function induced by immunotherapy increased the therapeutic efficacy of ICI by favoring systemic translocation of microbes and microbial metabolites that act through innate immune pathways contribute to the therapeutic success of immunotherapy. ${ }^{11}$ It has been found that patients who experience some grade of intestinal inflammation had a significantly higher chance of responding to ICI treatment. ${ }^{49}$ Studies in mice and humans with melanoma have shown that members of the Bacteroidales order were instrumental for the T helper 1 (Th1) antitumor immunity elicited by antiCTLA-4. ${ }^{8}$ Paradoxically, however, transplantation of Th1promoting Bacteroides fragilis was associated with a reduced deterioration of the enteric mucosa, ${ }^{33}$ thus segregating its role in immunotherapeutic effectiveness from that in toxicity. Our results are consistent with this observation as 3-IAld supplementation during immunotherapy, while strengthening the mucosal barrier function, did not impede the recruitment of $\mathrm{CD} 4^{+}$and $\mathrm{CD} 8^{+}$lymphocytes at the tumor site. Thus, the therapeutic success of anti-CTLA-4 antibody may occur independently of bacteria translocation and accumulation at the tumor site and likely occurs through alternative mechanisms, including the systemic translocation of metabolites, as recently proposed. ${ }^{11}$ In this regard, we found that 3-IAld itself, which is systemically detected after enteric administration, ${ }^{50}$ did not show a direct antitumor activity in vivo or in vitro on cultured B16 cells (data not shown), a finding consistent with the disparate role of AhR ligands in cancer. ${ }^{51}$ Thus, metabolites other than 3-IAld may contribute to the antitumor activity of CTLA-4 inhibitors in our setting. This is likely to occur as we have found that the levels of n-butyric and valeric acid were significantly increased after 3-IAld's treatment. That indoles are able to induce butyrate, known to promote IL-22 production ${ }^{52}$ while repressing IDO1 expression in the gut ${ }^{53}$ has already been reported. ${ }^{41}$ However, while protecting from intestinal epithelial damage, systemic butyrate limited the antitumor effect of CTLA-4 blockade in hosts with cancer. ${ }^{54}$ Neither the antitumor activity of anti-CTLA-4 antibody was impaired nor the serum levels of butyrate increased in 3-IAld-treated mice, as opposed to butyrate-treated mice (data not shown), a finding pointing to the potential clinical utility of 3-IAld in finely regulating the local versus systemic butyrate levels. Of interest, we found that valeric acid, a potent histone deacetylase inhibitor endowed with antitumor activity ${ }^{55}$ was increased after 3-IAld treatment. Although an attractive working hypothesis, whether and how the valeric acid contributes to the overall effects of 3-IAld is presently unknown.

It is clear that 3-IAld modulates the composition and function of the gut microbiome, an activity consistent with the ability of indole and indolyl compounds to act as transkingdom signaling molecules. ${ }^{56}$ We have indeed shown that 3-IAld exhibited potent antimicrobial activity in vitro ${ }^{57}$ and modulated the composition of airway and gut microbiota in 
A

$\mathrm{B}$

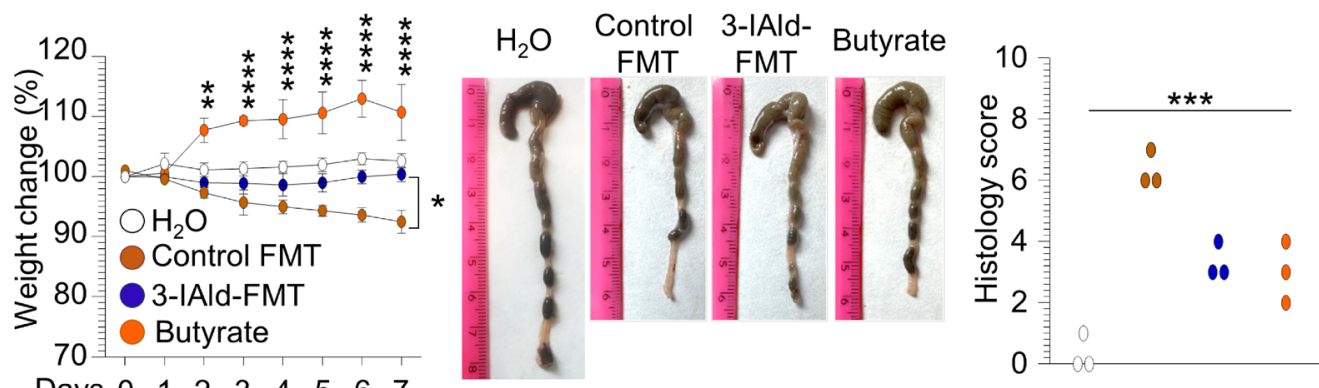

Days $\begin{array}{lllllllll}1 & 2 & 3 & 4 & 5 & 6 & 7\end{array}$

$\mathrm{D}$
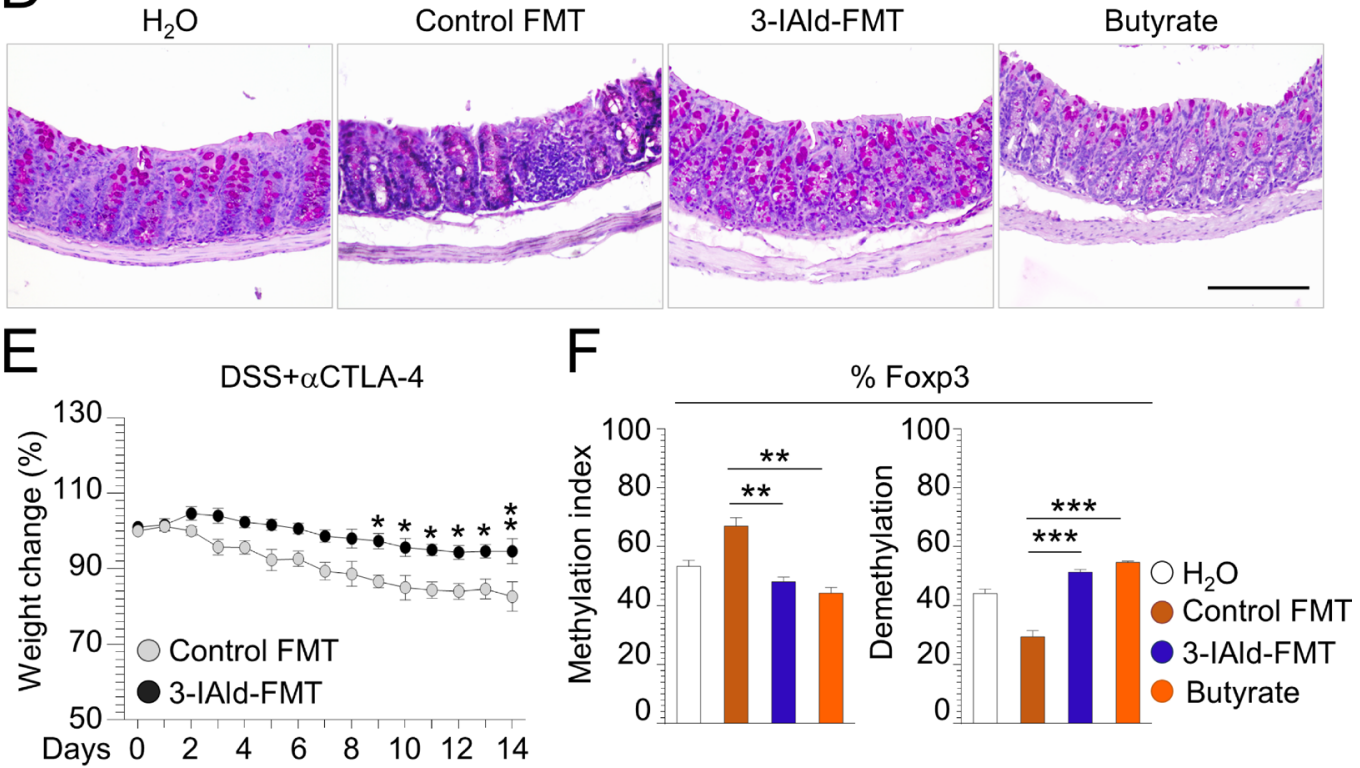

G

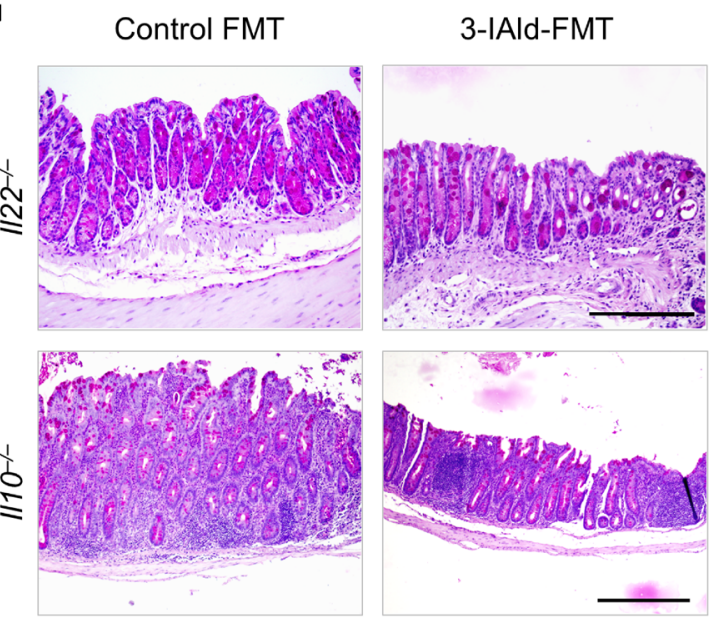

$\mathrm{H}$

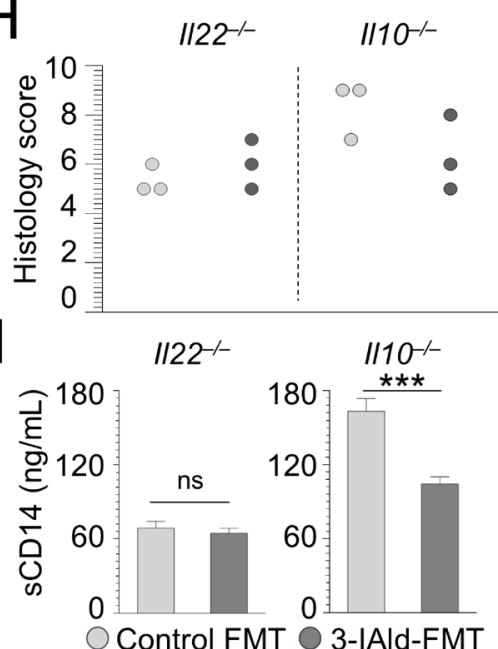

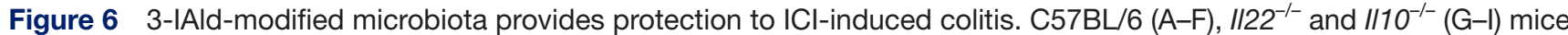
were subjected to DSS-colitis with (E) or without (A-D,F-I) anti-CTLA-4 and transplanted with fresh fecal pellets from control or 3 -IAld-treated mice 1 day before and 2 days after colitis induction. One group of mice was treated with $1 \%$ butyrate in drinking water 1 week before starting DSS administration. Mice were sacrificed 7 (A-D,F-I) or 14 (E) days after colitis induction and evaluated for (A,E) \% weight change, (B) gross pathology, (C,H) histology score, (D,G) colon histopathology (periodic acid-Schiff staining), (F) methylation/demethylation status of Foxp3 promoter in mesenteric lymph nodes, and (I) sCD14 serum levels. Photographs were taken with a high-resolution microscope (Olympus BX51), $\times 10$ and $\times 20$ magnification (scale bars, 500 and $200 \mu \mathrm{m})$. For histology, data are representative of three independent experiments. Each in vivo experiment includes 3 mice per group (6-12 mice in each experiment). Data are represented as mean \pm SD. Treated versus control FMT mice. One-way analysis of variance, Bonferroni post hoc test, (C) Kruskal-Wallis test. $\mathrm{H}_{2} \mathrm{O}$, untreated mice. ${ }^{*} \mathrm{P}<0.05$, ${ }^{\star \star} \mathrm{P}<0.01$, ${ }^{* \star *} \mathrm{P}<0.001$, ${ }^{* \star \star *} \mathrm{P}<0.0001$. 3-IAld, indole-3-carboxaldehyde; FMT, fecal microbiota transplantation. ns, not significant. 
A

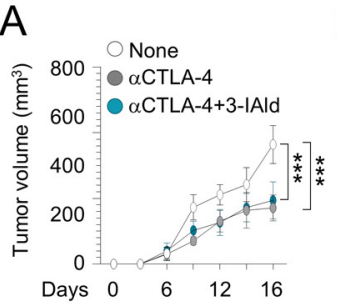

D

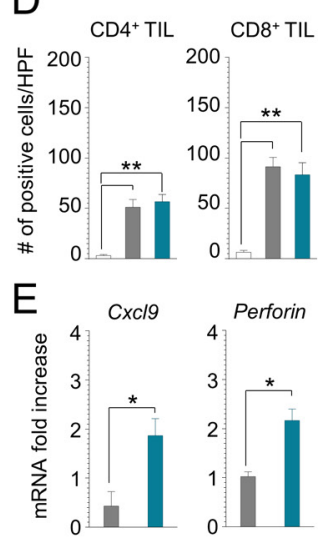

$\mathrm{F}$
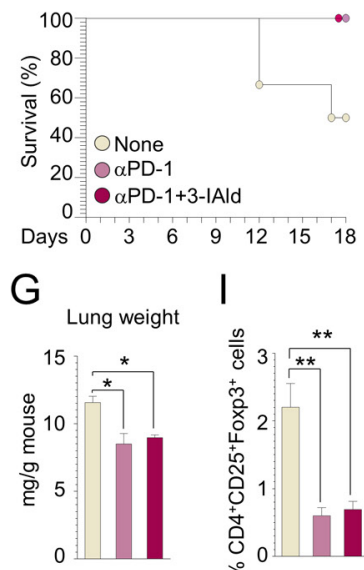

B

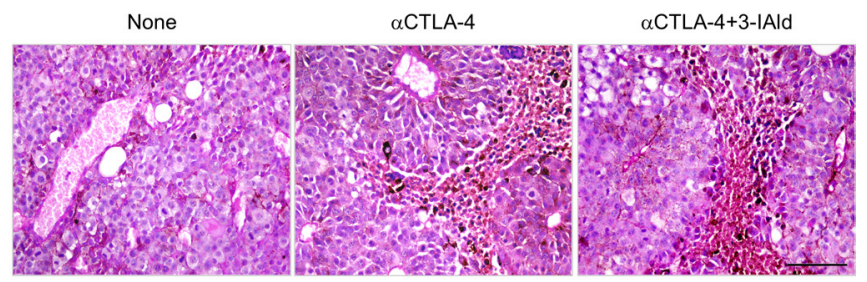

C

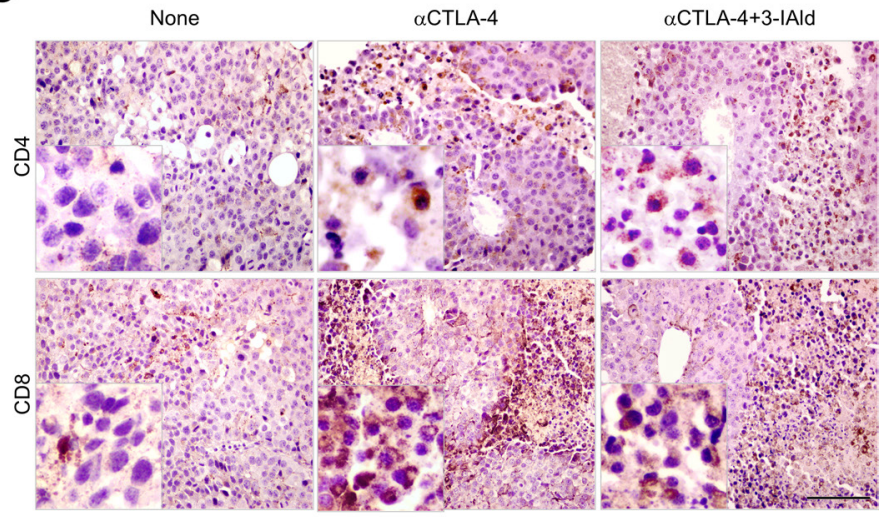

$\mathrm{H}$

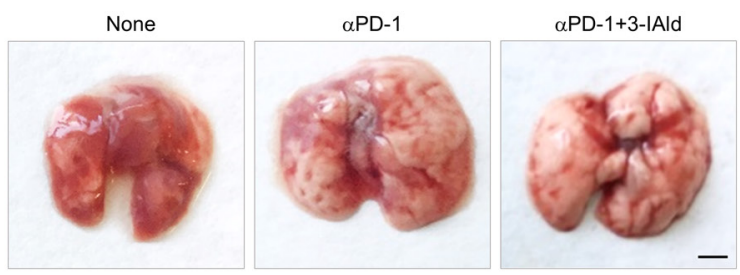

$J$

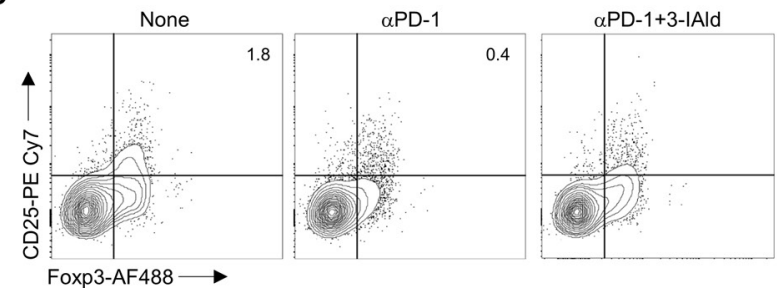

Figure 7 3-IAld does not interfere with the antitumor activity of anti-CTLA-4 antibody. (A-E) C57BL/6 mice were subcutaneously injected with B16 tumor cells and administered $100 \mu \mathrm{g}$ anti-CTLA-4 mAb or isotype control intraperitoneally four times at 3-day intervals up to 16 days. 3-IAld was administered intragastrically every other day. Mice were evaluated for (A) tumor growth, (B) histology (periodic acid-Schiff staining), (C) immunohistochemistry for $\mathrm{CD}^{+}$and $\mathrm{CD} 4^{+}$tumor-infiltrating cells and (D) number of positive cells per HPF, and (E) Cxcl9 and Perforin expression in melanoma. (F-J) C57BL/6 mice were orthotopically injected with LLC cells and administered $200 \mu \mathrm{g}$ anti-PD-1 mAb or isotype control intraperitoneally five times at 3-day intervals up to 18 days. 3-IAld was administered intragastrically every other day. Mice were evaluated for (F) \% survival, (G) lung weight, (H) lung gross pathology, (I, J) $\mathrm{CD} 4^{+} \mathrm{CD} 25^{+} \mathrm{Foxp} 3^{+}$cells. Photographs were taken with a high-resolution microscope (Olympus BX51), ×40 magnification (scale bars, $100 \mu \mathrm{m}$ ). For histology, data are representative of two independent experiments. Each in vivo experiment includes three mice per group (nine mice in each experiment). Data are represented as mean \pm SEM. Treated versus none (isotype control) or $\alpha$ CTLA-4/ $\alpha$ PD-1+3-IAld-treated versus $\alpha C T L A-4 / \alpha P D-1$-treated mice. One-way analysis of variance, Bonferroni post hoc test. ${ }^{\star} \mathrm{P}<0.05,{ }^{\star \star} \mathrm{P}<0.01,{ }^{{ }^{\star \star} \mathrm{P}}<0.001$. 3 -IAld, indole-3-carboxaldehyde; HPF, high-power field; TIL, tumor-infiltrating lymphocyte.

murine model of cystic fibrosis. ${ }^{58}$ Alternatively, 3-IAld may affect the microbiota composition via the regulatory activity of IL-22 on the microbiota. ${ }^{45}$ This mechanism has been recently reported for indole-3-carbinol that, by inducing IL-22 from ILC type 3, promoted the expansion of butyrateproducing bacteria, such as Roseburia spp. ${ }^{41}$ Consistent with the ability of 3-IAld to promote ILC3 (16), we obtained similar results here by showing the predominant expansion of sugar-degrading members of Bacteroidota and Firmicutes by 3-IAld. While Bacteroidota are involved in multiple activities, in addition to monosaccharide metabolism, Firmicutes rely on the PTS system for transport and metabolism of disaccharides, monosaccharides and other sugar derivatives,$^{59}$ eventually converging on the butyrate biosynthesis pathway and butyrate production. ${ }^{60}$ Growing experimental evidences support the importance of butyrate, a four-carbon 
SCFA, for intestinal health, ${ }^{61}$ even though diverging results were obtained on butyrate induction or supplementation in patients with inflammatory bowel disease.$^{62}$ Transplantation of feces from 3-IAld-treated mice or butyrate supplementation was able to protect from colitis in IL-22-sufficient mice, thus directly proving that 3-IAld at the forefront of hostmicrobe interactions coordinates the activity of the microbiota and the host during ICI therapy. Likely as a result of the cross-feeding networks, we also found that sulfate reducing bacteria belonging to the Desulfobacterota phylum, abundantly present in the human gut, ${ }^{63}$ were expanded after 3-IAld treatment. It is known that dietary components provide fiber and macromolecules that are degraded by bacterial enzymes to monomers, and these are fermented by intestinal bacteria with the production to molecular hydrogen which promotes the metabolic dominance by sulfate-reducing bacteria. ${ }^{64}$ As one of the three mammalian gasotransmitters, $\mathrm{H}_{2} \mathrm{~S}$ plays a major role in maintaining physiological homeostasis. Numerous homeostatic functions have been proposed for $\mathrm{H}_{2} \mathrm{~S}$, including cytoprotection and anti-inflammatory activity in the gut, neuromodulation, and cardiovascular function. ${ }^{65}$ Therefore, 3-IAld appears to modulate the function of the microbiota to engage multiple protective functions, mainly centered on carbohydrate metabolism, which raises the possibility of conceptualizing 3-IAld as a prebiotic substance.

Much has to be learned to fully harness gut microbiome for clinical applicability, including the definition of a shared gut microbiome across various solid tumors treated with ICI. Our study provides a piece of evidence that moving past bacterial phylogeny and focusing on bacterial metabolome may lead to a new class of discrete molecules that working at the interface between microbes and the host immune system may optimize ICI therapy via a precision enteric medicine that targets the epithelial barrier for mucosal homeostasis and prevention of distant immunopathology. ${ }^{66}$ Future human studies are needed to confirm whether 3-IAld, commonly detected in humans, could be used as a modifier of the mucosal milieu to provide an allied microbiota to optimize ICI regimens and/or as a biomarker for predicting ICI efficacy. In this regard, it is encouraging that 3-IAld attenuated the increase in epithelial permeability caused by inflammatory stimuli in an in vitro preclinical screen using the human Caco-2 epithelial cell line. ${ }^{67}$

Contributors Conceptualization: GR, FA, LR, and CC; methodology: GR, $\mathrm{MPa}, \mathrm{MMB}, \mathrm{FD}$ '0, IS, CS, FR, CA, MG, and OB; formulation of 3-IAld for oral administration: MR, MPu, and SG; mass spectrometry: GP; metagenomics analysis: EN; writing —original draft preparation and review and editing: GR, CC, and LR; supervision and funding acquisition: LR. LR acts as the guarantor for the overall content. All authors have read and agreed to the published version of the manuscript.

Funding This work was supported by MicroTher (ERC-2018-PoC-813099, to LR).

Competing interests None declared.

Patient consent for publication Not applicable.

Ethics approval All animal care and experimental protocols were performed according to Italian Approved Animal Welfare Authorization 360/2015-PR and Legislative Decree 26/2014 regarding the animal license obtained by the Italian Ministry of Health lasting for 5 years (2015-2020).

Provenance and peer review Not commissioned; externally peer reviewed.
Data availability statement Data sharing was not applicable as no datasets were generated and/or analyzed for this study. Data are available on reasonable request.

Supplemental material This content has been supplied by the author(s). It has not been vetted by BMJ Publishing Group Limited (BMJ) and may not have been peer-reviewed. Any opinions or recommendations discussed are solely those of the author(s) and are not endorsed by BMJ. BMJ disclaims all liability and responsibility arising from any reliance placed on the content. Where the content includes any translated material, BMJ does not warrant the accuracy and reliability of the translations (including but not limited to local regulations, clinical guidelines, terminology, drug names and drug dosages), and is not responsible for any error and/or omissions arising from translation and adaptation or otherwise.

Open access This is an open access article distributed in accordance with the Creative Commons Attribution Non Commercial (CC BY-NC 4.0) license, which permits others to distribute, remix, adapt, build upon this work non-commercially, and license their derivative works on different terms, provided the original work is properly cited, appropriate credit is given, any changes made indicated, and the use is non-commercial. See http://creativecommons.org/licenses/by-nc/4.0/.

\section{ORCID iDs}

Giorgia Renga http://orcid.org/0000-0002-9762-6493

Giuseppe Pieraccini http://orcid.org/0000-0003-3103-3924

\section{REFERENCES}

1 Schoenfeld AJ, Hellmann MD. Acquired resistance to immune checkpoint inhibitors. Cancer Cell 2020;37:443-55.

2 Choi J, Lee SY. Clinical characteristics and treatment of immunerelated adverse events of immune checkpoint inhibitors. Immune Netw 2020;20:e9.

3 Bauché D, Mauze S, Kochel C, et al. Antitumor efficacy of combined CTLA4/PD-1 blockade without intestinal inflammation is achieved by elimination of Fc $\gamma R$ interactions. J Immunother Cancer 2020;8.

4 Pitt JM, Vétizou M, Daillère R, et al. Resistance mechanisms to immune-checkpoint blockade in cancer: tumor-intrinsic and -Extrinsic factors. Immunity 2016;44:1255-69.

5 Roy S, Trinchieri G. Microbiota: a key orchestrator of cancer therapy. Nat Rev Cancer 2017;17:271-85.

6 lida N, Dzutsev A, Stewart CA, et al. Commensal bacteria control cancer response to therapy by modulating the tumor microenvironment. Science 2013;342:967-70.

7 Viaud S, Saccheri F, Mignot G, et al. The intestinal microbiota modulates the anticancer immune effects of cyclophosphamide. Science 2013;342:971-6.

8 Vétizou M, Pitt JM, Daillère R, et al. Anticancer immunotherapy by CTLA-4 blockade relies on the gut microbiota. Science 2015;350:1079-84.

9 Gopalakrishnan V, Spencer CN, Nezi L, et al. Gut microbiome modulates response to anti-PD-1 immunotherapy in melanoma patients. Science 2018;359:97-103.

10 Andrews MC, Duong CPM, Gopalakrishnan V, et al. Gut microbiota signatures are associated with toxicity to combined CTLA-4 and PD1 blockade. Nat Med 2021;27:1432-41.

11 Almonte AA, Rangarajan H, Yip D, et al. How does the gut microbiome influence immune checkpoint blockade therapy? Immunol Cell Biol 2021;99:361-72.

12 Yi M, Jiao D, Qin S, et al. Manipulating gut microbiota composition to enhance the therapeutic effect of cancer immunotherapy. Integr Cancer Ther 2019;18:1534735419876351.

13 Malczewski AB, Navarro S, Coward JI, et al. Microbiome-derived metabolome as a potential predictor of response to cancer immunotherapy. J Immunother Cancer 2020;8.

14 Agus A, Planchais J, Sokol H. Gut microbiota regulation of tryptophan metabolism in health and disease. Cell Host Microbe 2018;23:716-24.

15 Stockinger B, Shah K, Wincent E. AHR in the intestinal microenvironment: safeguarding barrier function. Nat Rev Gastroenterol Hepatol 2021;18:559-70.

16 Zelante T, lannitti RG, Cunha C, et al. Tryptophan catabolites from microbiota engage aryl hydrocarbon receptor and balance mucosal reactivity via interleukin-22. Immunity 2013;39:372-85.

17 Dong F, Hao F, Murray IA, et al. Intestinal microbiota-derived tryptophan metabolites are predictive of Ah receptor activity. Gut Microbes 2020;12:1-24.

18 Hubbard TD, Murray IA, Perdew GH. Indole and tryptophan metabolism: endogenous and dietary routes to Ah receptor activation. Drug Metab Dispos 2015;43:1522-35. 
19 Anesi A, Rubert J, Oluwagbemigun K, et al. Metabolic profiling of human plasma and urine, targeting tryptophan, tyrosine and branched chain amino acid pathways. Metabolites 2019:9:metabo9110261. doi:10.3390/metabo9110261

20 Vyhlídalová B, Krasulová K, Pečinková P, et al. Gut microbial catabolites of tryptophan are ligands and agonists of the aryl hydrocarbon receptor: a detailed characterization. Int $\mathrm{J} \mathrm{Mol} \mathrm{Sci}$ 2020;21 doi:10.3390/ijms21072614

21 Costantini C, Nunzi E, Spolzino A, et al. Pharyngeal microbial signatures are predictive of the risk of fungal pneumonia in hematologic patients. Infect Immun 2021;89:e0010521.

22 Kang KY, Lee SH, Jung SM, et al. Downregulation of Tryptophanrelated metabolomic profile in rheumatoid arthritis synovial fluid. $J$ Rheumatol 2015;42:2003-11.

23 Yu J, Luo Y, Zhu Z, et al. A tryptophan metabolite of the skin microbiota attenuates inflammation in patients with atopic dermatitis through the aryl hydrocarbon receptor. J Allergy Clin Immunol 2019;143:e12:2108-19.

24 Rothhammer V, Mascanfroni ID, Bunse L, et al. Type I interferons and microbial metabolites of tryptophan modulate astrocyte activity and central nervous system inflammation via the aryl hydrocarbon receptor. Nat Med 2016;22:586-97.

25 Alexeev EE, Lanis JM, Kao DJ, et al. Microbiota-Derived indole metabolites promote human and murine intestinal homeostasis through regulation of interleukin-10 receptor. Am J Pathol 2018:188:1183-94.

26 Zelante T, Puccetti M, Giovagnoli S, et al. Regulation of host physiology and immunity by microbial indole-3-aldehyde. Curr Opin Immunol 2021;70:27-32.

27 Puccetti M, Pariano M, Borghi M, et al. Enteric formulated indole3-carboxaldehyde targets the aryl hydrocarbon receptor for protection in a murine model of metabolic syndrome. Int $J$ Pharm 2021;15:120610.

28 Renga G, Bellet MM, Pariano M, et al. Thymosin $\alpha 1$ protects from CTLA-4 intestinal immunopathology. Life Sci Alliance 2020;3:e202000662. doi:10.26508/lsa.202000662

29 Powrie F, Leach MW, Mauze S, et al. Inhibition of Th1 responses prevents inflammatory bowel disease in SCID mice reconstituted with CD45RBhi CD4+ T cells. Immunity 1994;1:553-62.

30 Puccetti M, Giovagnoli S, Zelante T, et al. Development of novel indole-3-aldehyde-loaded gastro-resistant spray-dried microparticles for postbiotic small intestine local delivery. J Pharm Sci 2018;107:2341-53

31 Singh NP, Singh UP, Singh B, et al. Activation of aryl hydrocarbon receptor (AhR) leads to reciprocal epigenetic regulation of FOXP3 and IL-17 expression and amelioration of experimental colitis. PLoS One 2011;6:e23522.

$32 \mathrm{He} \mathrm{Y,} \mathrm{Li} \mathrm{X,} \mathrm{Yu} \mathrm{H,} \mathrm{et} \mathrm{al.} \mathrm{The} \mathrm{functional} \mathrm{role} \mathrm{of} \mathrm{fecal} \mathrm{microbiota}$ transplantation on dextran sulfate sodium-induced colitis in mice. Front Cell Infect Microbiol 2019;9:393.

33 Wang F, Yin Q, Chen L, et al. Bifidobacterium can mitigate intestinal immunopathology in the context of CTLA-4 blockade. Proc Natl Acad Sci U S A 2018:115:157-61.

34 Perez-Ruiz E, Minute L, Otano I, et al. Prophylactic TNF blockade uncouples efficacy and toxicity in dual CTLA-4 and PD-1 immunotherapy. Nature 2019;569:428-32.

35 Yu X, Wang Y, Deng M, et al. The basic leucine zipper transcription factor NFIL3 directs the development of a common innate lymphoid cell precursor. Elife 2014;3 doi:10.7554/eLife.04406

36 Schulz-Kuhnt A, Neurath MF, Wirtz S, et al. Innate lymphoid cells as regulators of epithelial integrity: therapeutic implications for inflammatory bowel diseases. Front Med 2021;8:656745.

37 Dhar P, McAuley J. The role of the cell surface mucin MUC1 as a barrier to infection and regulator of inflammation. Front Cell Infect Microbiol 2019;9:117.

38 Tabung FK, Birmann BM, Epstein MM, et al. Influence of dietary patterns on plasma soluble CD14, a surrogate marker of gut barrier dysfunction. Curr Dev Nutr 2017;1:e001396. doi:10.3945/ cdn.117.001396

39 Grohmann U, Fallarino F, Puccetti P. Tolerance, DCs and tryptophan: much ado about IDO. Trends Immunol 2003;24:242-8.

40 Kühn R, Löhler J, Rennick D, et al. Interleukin-10-deficient mice develop chronic enterocolitis. Cell 1993;75:263-74.

41 Busbee PB, Menzel L, Alrafas HR, et al. Indole-3-carbinol prevents colitis and associated microbial dysbiosis in an IL-22-dependent manner. JCl Insight 2020;5:127551. doi:10.1172/jci.insight.127551
42 Qiu J, Heller JJ, Guo X, et al. The aryl hydrocarbon receptor regulates gut immunity through modulation of innate lymphoid cells. Immunity 2012;36:92-104.

43 Wei H-X, Wang B, Li B. IL-10 and IL-22 in mucosal immunity: driving protection and pathology. Front Immunol 2020;11:1315.

44 Dong F, Perdew GH. The aryl hydrocarbon receptor as a mediator of host-microbiota interplay. Gut Microbes 2020;12:1859812.

45 Zenewicz LA, Yin X, Wang G, et al. IL-22 deficiency alters colonic microbiota to be transmissible and colitogenic. J Immunol 2013;190:5306-12.

46 Dongowski G, Lorenz A, Anger H. Degradation of pectins with different degrees of esterification by Bacteroides thetaiotaomicron isolated from human gut flora. Appl Environ Microbiol 2000;66:1321-7.

47 Tamanai-Shacoori Z, Smida I, Bousarghin L, et al. Roseburia spp.: a marker of health? Future Microbiol 2017;12:157-70. doi:10.2217/ fmb-2016-0130

48 Hodi FS, O'Day SJ, McDermott DF, et al. Improved survival with ipilimumab in patients with metastatic melanoma. N Engl $J$ Med 2010;363:711-23.

49 Soularue E, Lepage P, Colombel JF, et al. Enterocolitis due to immune checkpoint inhibitors: a systematic review. Gut 2018:67:2056-67.

50 Puccetti M, Pariano M, Borghi M, et al. Enteric formulated indole3-carboxaldehyde targets the aryl hydrocarbon receptor for protection in a murine model of metabolic syndrome. Int $\mathrm{J}$ Pharm 2021;602:120610.

51 Paris A, Tardif N, Galibert M-D, et al. AhR and cancer: from gene profiling to targeted therapy. Int J Mol Sci 2021;22 doi:10.3390/ ijms22020752

52 Yang W, Yu T, Huang X, et al. Intestinal microbiota-derived shortchain fatty acids regulation of immune cell IL-22 production and gut immunity. Nat Commun 2020;11:4457.

53 Martin-Gallausiaux C, Larraufie P, Jarry A, et al. Butyrate Produced by Commensal Bacteria Down-Regulates Indolamine 2,3-Dioxygenase 1 (IDO-1) Expression via a Dual Mechanism in Human Intestinal Epithelial Cells. Front Immunol 2018;9:2838.

54 Coutzac C, Jouniaux J-M, Paci A, et al. Systemic short chain fatty acids limit antitumor effect of CTLA-4 blockade in hosts with cancer. Nat Commun 2020;11:2168.

55 Han R, Nusbaum O, Chen X, et al. Valeric acid suppresses liver cancer development by acting as a novel HDAC inhibitor. Mol Ther Oncolytics 2020;19:8-18.

56 Lee J-H, Wood TK, Lee J. Roles of indole as an interspecies and interkingdom signaling molecule. Trends Microbiol 2015;23:707-18.

57 Puccetti M, Xiroudaki S, Ricci M, et al. Postbiotic-enabled targeting of the host-microbiota-pathogen interface: hints of antibiotic decline? Pharmaceutics 2020;12:1. doi:10.3390/pharmaceutics12070624

58 Puccetti M, Pariano M, Renga G, et al. Targeted drug delivery technologies potentiate the overall therapeutic efficacy of an indole derivative in a mouse cystic fibrosis setting. Cells 2021;10:1601. doi:10.3390/cells10071601

59 Saier $\mathrm{MH}$. The bacterial phosphotransferase system: new frontiers 50 years after its discovery. J Mol Microbiol Biotechnol 2015;25:73-8.

60 Tanca A, Abbondio M, Palomba A, et al. Potential and active functions in the gut microbiota of a healthy human cohort. Microbiome 2017;5:79.

61 Canani RB, Costanzo MD, Leone L, et al. Potential beneficial effects of butyrate in intestinal and extraintestinal diseases. World $J$ Gastroenterol 2011;17:1519-28.

62 Magnusson MK, Isaksson S, Öhman L. The anti-inflammatory immune regulation induced by butyrate is impaired in inflamed intestinal mucosa from patients with ulcerative colitis. Inflammation 2020;43:507-17.

63 Chen Y-R, Zhou L-Z, Fang S-T, et al. Isolation of Desulfovibrio spp. from human gut microbiota using a next-generation sequencing directed culture method. Lett Appl Microbiol 2019;68:553-61.

64 Barton LL, Ritz NL, Fauque GD, et al. Sulfur cycling and the intestinal microbiome Dig Dis Sci 2017:62:2241-57.

65 Carbonero F, Benefiel AC, Alizadeh-Ghamsari AH, et al. Microbial pathways in colonic sulfur metabolism and links with health and disease. Front Physiol 2012;3:448.

66 Akdis CA. Does the epithelial barrier hypothesis explain the increase in allergy, autoimmunity and other chronic conditions? Nat Rev Immunol 2021;21:739-51.

67 Scott SA, Fu J, Chang PV. Microbial tryptophan metabolites regulate gut barrier function via the aryl hydrocarbon receptor. Proc Natl Acad Sci U S A 2020;117:19376-87. 